Journal for ImmunoTherapy of Cancer

\title{
Overcoming tumor heterogeneity by ex vivo arming of $T$ cells using multiple bispecific antibodies
}

\author{
Jeong A Park (D) , ${ }^{1,2}$ Nai-Kong V Cheung (D) ${ }^{1}$
}

To cite: Park JA, Cheung N$\mathrm{KV}$. Overcoming tumor heterogeneity by ex vivo arming of $T$ cells using multiple bispecific antibodies. Journal for ImmunoTherapy of Cancer 2022;10:e003771. doi:10.1136/ jitc-2021-003771

- Additional supplemental material is published online only. To view, please visit the journal online (http://dx.doi.org/10. 1136/jitc-2021-003771).

Accepted 22 December 2021

Check for updates

(C) Author(s) (or their employer(s)) 2022. Re-use permitted under CC BY-NC. No commercial re-use. See rights and permissions. Published by BMJ.

${ }^{1}$ Department of Pediatrics, Memorial Sloan Kettering Cancer Center, New York, New York, USA

${ }^{2}$ Department of Pediatrics, Inha University Hospital, Incheon, Republic of Korea

Correspondence to Dr Nai-Kong V Cheung; cheungn@mskcc.org

\section{ABSTRACT}

Background Tumorous heterogeneity is a hallmark of tumor evolution and cancer progression, being a longstanding challenge to targeted immunotherapy. Ex vivo armed T cells (EATs) using IgG-(L)-scFv bispecific antibodies (BsAbs) are potent tumor-specific cytotoxic effectors. To improve the anti-tumor efficacy of EATs against heterogeneous solid tumors, we explored multiantigen targeting approaches.

Methods Ex vivo expanded T cells were armed with BsAbs built on the IgG-(L)-scFv platform, where an antiCD3 (huOKT3) scFv was attached to the carboxyl end of both light chains of a tumor specific IgG. Multispecificity was created by combining monospecific EATs, combining BsAbs on the same T cell, or combining specificities on the same antibody. Three multi-antigens targeting EAT strategies were tested: (1) pooled-EATs (EATs each with unique specificity administered simultaneously) or alternate-EATs (EATs each with unique specificity administered in an alternating schedule), (2) dual-EATs or multi-EATs (T cells simultaneously armed with $\geq 2$ BsAbs), and (3) TriAb-EATs (T cells armed with BsAb specific for two targets besides CD3 (TriAb)). The properties and efficiencies of these three strategies were evaluated by flow cytometry, in vitro cytotoxicity, cytokine release assays, and in vivo studies performed in BALB-Rag $2^{/-} \mathrm{IL}-$ 2R- $\gamma c-\mathrm{KO}$ (BRG) mice xenografted with cancer cell line (CDX) or patient-derived tumor (PDX).

Results Multi-EATs retained target antigen specificity and anti-tumor potency. Cytokine release with multi-EATs in the presence of tumor cells was substantially less than when multiple BsAbs were mixed with unarmed T cells. When tested against CDXs or PDXs, dual-EATs or multiEATs effectively suppressed tumor growth without clinical toxicities. Most importantly, dual-EATs or multi-EATs were highly efficient in preventing clonal escape while monoEATs or TriAb- EATs were not as effective.

Conclusions Multi-EATs have the potential to increase potency, reduce toxicity, and overcome tumor heterogeneity without excessive cytokine release. Arming T cells with multiple BsAbs deserves further exploration to prevent or to treat cancer resistance.

\section{INTRODUCTION}

Immunotherapy based on retargeting $\mathrm{T}$ cells has renewed hope for durable cancer cure. However, success has largely been limited to hematological malignancies and a few cancers with high tumor mutational burden. Treatment-related adverse events including cytokine release syndrome, neurotoxicity, and long-term on-target off-tumor toxicities, particularly for targets expressed in normal tissues (eg, HER2 ${ }^{1}$ ), are major challenges, hampering clinical applications. For solid tumors, additional hurdles have emerged, such as tumorous heterogeneity, physical barriers, and immunosuppressive tumor microenvironment (TME). ${ }^{2}$ Even for hematologic malignancies highly susceptible to $\mathrm{T}$ cell immunotherapy, tumor-associated antigens (TAAs) are often heterogeneous and prone to downregulation or loss, whereby initial responses are not durable and followed by tumor escape and treatment failure. ${ }^{3-5}$ To overcome tumor heterogeneity encountered by engineered $\mathrm{T}$ cells in solid tumors, increasing specificity to two or more targets has not been adequately explored.

$\mathrm{T}$ cell engaging bispecific antibodies (abbreviated as BsAbs) have demonstrated promising antitumor efficacy in both hematologic malignancies (Blinatumomab, anti-CD19 $\mathrm{x}$ anti-CD3) ${ }^{6}$ and solid tumors (Catumaxomab, anti-EpCAM $\times$ anti-CD3). ${ }^{78}$ Yet despite decades of research and development, only these two BsAbs were clinically approved for cancer treatment. Most BsAbs have failed due to insufficient potency or dose-limiting toxicities that were typically cytokine-related. T cells armed with chemically conjugated anti-GD2 $\times$ anti-CD3 (hu3F8 $x$ mouse OKT3 (NCT02173093)), anti-HER2 $\times$ anti-CD3 (trastuzumab $\times$ mouse OKT3 $($ NCT00027807)), or anti-EGFR $\times$ anti-CD3 (cetuximab $\times$ mouse OKT3 (NCT04137536)), have proven safe in multiple clinical trials without cytokine storm, neurotoxicity, or long-term major organ toxicities. ${ }^{9-12}$ Recent structure function analyses of BsAbs have shown that $\mathrm{T}$ cells armed ex vivo with IgG-(L)scFv platformed BsAb (ex vivo armed T cells, EATs) were highly effective against a variety of 
cancers when compared with those armed with other standard formats of BsAb including chemical conjugates. ${ }^{13-15}$ Target antigen-specific EATs effectively infiltrated into tumors despite tissue barriers and immune hostile TME, exerting potent and durable antitumor response. To overcome tumor antigen heterogeneity, we now explore multiantigen targeting approaches. Multispecificity was created by combining monospecific EATs, combining multiple BsAbs on the same T cell, or combining specificities on the same antibody. We now test the following EAT strategies in vitro and in vivo: (1) pooled-EATs (simultaneous combination of monospecific EATs) or alternateEATs (alternating combination of monospecific EATs), (2) dual- or multi-EATs ( $\mathrm{T}$ cells armed simultaneously with $\geq 2$ BsAbs), and (3) TriAb-EATs (T cells armed with BsAb specific for two different tumor targets beside CD3 $(\operatorname{TriAb}))$.

\section{METHODS}

\section{Ex vivo $\mathrm{T}$ cell activation and arming with $\mathrm{BsAb}$}

Peripheral blood mononuclear cells (PBMCs) were separated from buffy coats (New York Blood Center) using Ficoll. The naive $\mathrm{T}$ cells were purified from human PBMC using Pan T cell isolation kit (Miltenyi Biotec, Cat\#130096535) and activated and expanded by CD3/ CD28 Dynabeads (Gibco, Cat\#11 132D) for 7 to 14 days in the presence of $30 \mathrm{IU} / \mathrm{mL}$ of IL-2 according to manufacturer's instructions. T cells were harvested between day 7 and day 14 (median day 10) and, unless stated otherwise, these cultured $\mathrm{T}$ cells were used for arming or all $\mathrm{T}$ cell experiments. EATs were generated by incubating $\mathrm{T}$ cells with $\mathrm{BsAb}$ for $20 \mathrm{~min}$ at room temperature. After incubation, these T cells were washed with PBS twice. The T cell number administered per dose was $2 \times 10^{7}$ cells based on previous reports ${ }^{16}$ with supplementary subcutaneous IL-2 (1000 IU).

\section{Quantification of BsAb bound on EAT}

These ex vivo BsAb armed T cells (EATs) were tested for cell surface density of BsAb (MFI) using APC anti-human IgG Fc antibody (Rat IgG2a, א), (BioLegend, Cat\# 410712, RRID:AB_2565790). The MFIs were referenced to antibody binding capacity $(\mathrm{ABC})$ using anti-rat quantum beads Quantum Simply Cellular microspheres (Bio-Rad, Cat\# FCSC815A, RRID:AB_10061915) for quantification of BsAb bound on T cell. All samples were triplicates, and the experiments were repeated more than twice to ensure consistency.

\section{Bispecific antibody}

All BsAbs were synthesized as previously described (US patent\#62/896415) ${ }^{17-20}$ For each BsAb, scFv of huOKT3 was fused to the C-terminus of the light chain of human IgG1 via a C-terminal (G4S) 3 linker. ${ }^{21}$ N297A and K322A on Fc were generated with site-directed mutagenesis via primer extension in polymerase chain reactions. ${ }^{22}$ The nucleotide sequence encoding each BsAb was synthesized by GenScript and subcloned into a mammalian expression vector. Each BsAb was produced using Expi293 expression system (Thermo Fischer Scientific) separately. Antibodies were purified with protein A affinity column chromatography. The purity of BsAbs was evaluated by size-exclusion high performance liquid chromatography and showed high levels of purity $(>90 \%)$. The BsAbs remained stable after multiple freeze-thaw cycles. Biochemistry data of the BsAbs used in this study were summarized in online supplemental table $\mathrm{S} 1 .^{1314}$

\section{Tumor cell lines}

Neuroblastoma cell line, IMR-32 (ATCC Cat\# CCL-127, RRID:CVCL_0346), osteosarcoma cell line, 143B (ATCC Cat\# CRL-8303, RRID:CVCL_2270), primitive neuroectodermal tumor cell line TC-32 (RRID:CVCL-7151), breast cancer cell line HCC1954 (ATCC Cat\# CRL-2338, RRID:CVCL_1259), gastric cancer cell line NCI-N87 (ATCC Cat\# CRL-2338, RRID:CVCL_1259), acute monocytic leukemia (AML-M5a) cell line MOLM13 (DSMZ Cat\# ACC-554, RRID:CVCL_2119), prostate cancer cell line LNCaP-AR (ATCC Cat\# CRL-1740, RRID:CVCL_1379), and melanoma cell line M14 (NCI-DTP Cat\# M14, RRID:CVCL_1395) were used for experiments. All cancer cell lines were authenticated by short tandem repeats profiling using PowerPlex 1.2 System (Promega, Cat\# DC8942), and periodically tested for mycoplasma infection using a commercial kit (Lonza, Cat\# LT07-318). The luciferase-labeled melanoma cell line M14Luc, osteosarcoma cell line 143BLuc, and neuroblastoma cell line IMR32Luc were generated by retroviral infection with an SFG-GF Luc vector.

\section{Antibody-dependent T cell-mediated cytotoxicity}

EAT-mediated cytotoxicity was performed using ${ }^{51} \mathrm{Cr}$ release as described previously, ${ }^{19}$ and $\mathrm{EC}_{50}$ was calculated using SigmaPlot software. Target cell lines were cultured in RPMI-1640 (Cellgro) supplemented with 10\% fetal bovine serum (FBS, Life Technologies) and harvested with EDTA/Trypsin. These target cells were labeled with sodium ${ }^{51} \mathrm{Cr}$ chromate (Amersham, Arlington Height, IL) at $100 \mu \mathrm{Ci} / 10^{6}$ cells at $37^{\circ} \mathrm{C}$ for 1 hour. After washing twice, these radiolabeled target cells were plated in 96-well plates in triplicates. EATs were added to target cells at decreasing effector to target cell ratios (E:T ratios), at twofold dilutions from 10:1. After incubation at $37^{\circ} \mathrm{C}$ for 4 hours, the released ${ }^{51} \mathrm{Cr}$ was measured by a gamma counter (Packed Instrument, Downers Grove, IL). Percentage of specific lysis was calculated using the formula where cpm represented counts per minute of ${ }^{51} \mathrm{Cr}$ released.

$$
\frac{100 \% \times(\text { experimental cpm-background } c p m)}{(\text { total cpm-background cpm })}
$$

Total release of ${ }^{51} \mathrm{Cr}$ was assessed by lysis with $10 \%$ SDS (Sigma, St Louis, Mo) and background release was measured in the absence of effector cells and antibodies. These experiments were repeated more than twice using different $\mathrm{T}$ cell donors. 


\section{Cytokine release assays}

Human cytokine release by EATs or unarmed $\mathrm{T}$ cells mixed with BsAb was analyzed in vitro and in vivo. Human Th1 cell released cytokines were analyzed by LEGENDplexTM Human Th1 Panel (Biolegend, Cat\# 741035). Five human T cell cytokines including IL-2, IL-6, IL-10, IFN- $\gamma$ and TNF- $\alpha$ were analyzed after incubation with target antigen ( + ) tumor cell lines at $37^{\circ} \mathrm{C}$ for 4 hours (in vitro). Mouse serum cytokines were analyzed between 3 and 4 hours after each EAT injection, and 5 mice per group were analyzed. All values are representative of experiments performed in triplicate.

\section{In vivo experiments}

All animal experiments were performed in compliance with Memorial Sloan Kettering Cancer Center's institutional Animal Care and Use Committee guidelines. In vivo antitumor response was evaluated using CDXs or PDXs. Cancer cells suspended in Matrigel (Corning Corp, Tewksbury MA) or PDXs were implanted into the right flank of $6-10$ week-old BALB-Rag2 ${ }^{\prime}$ IL-2R- $\gamma c-$ KO (BRG) mice (Taconic Biosciences). ${ }^{23}$ The following cancer cell lines and cell doses were used: $1 \times 10^{6}$ of 143 BLuc, $5 \times 10^{6}$ of IMR32Luc, $5 \times 10^{6}$ of HCC1954, $5 \times 10^{6}$ of LNCaP-AR, and $5 \times 10^{6}$ of TC-32. For mixed lineage CDX, $2.5 \times 10^{6}$ of IMR32Luc and $2.5 \times 10^{6}$ of HCC1954 were mixed and implanted into each mouse. Three osteosarcoma, one Ewing sarcoma family of tumors (EFT), and one breast cancer PDXs were established from fresh surgical specimens with MSKCC IRB approval. To avoid biological variables, only female mice were used for in vivo experiments except LNCaP-AR CDXs using male mice. Treatment was initiated after tumors were established, average tumor volume of $100 \mathrm{~mm}^{3}$ when measured using TM900 scanner (Piera, Brussels, BE). Before treatment, mice with small tumors $\left(<50 \mathrm{~mm}^{3}\right)$ or infection signs were excluded from randomization to experimental groups. Tumor growth curves and overall survival was analyzed, and the overall survival was defined as the time from start of treatment to when tumor volume reached $2000 \mathrm{~mm}^{3}$. To define the well-being of mice, CBC analyses, body weight, general activity, physical appearance, and GVHD scoring were monitored. All animal experiments were repeated twice more. In each individual experiment, all $\mathrm{T}$ cells were derived from a single donor. More than two donors were used to ensure reproducibility and consistency among experiments.

\section{GD2 by fresh frozen tumor section staining}

Fresh frozen tumor sections were made using Tissue-Tek OCT (Miles Laboratories, Elkhart, IN) with liquid nitrogen and stored at $-80^{\circ} \mathrm{C}$. The tumor sections were stained with mouse IgG3 mAb 3F8 for GD2 as previously described. ${ }^{24}$ Stained slides were captured using a Nikon ECLIPSE Ni-U microscope and analyzed, and the tissue staining intensity and percentage of positive cells were compared with positive and negative controls. Each sample was assessed and graded by two independent observers.

\section{Immunohistochemistry for T cell infiltration and HER2 expression}

Harvested xenografts were formalin-fixed paraffinembedded (FFPE) and tested for immunohistochemistry (IHC). IHC staining was performed by Molecular Cytology Core Facility of MSKCC using Discovery XT processor (Ventana Medical Systems). FFPE tumor sections were deparaffinized with EZPrep buffer (Ventana Medical Systems), antigen retrieval was performed with CC1 buffer (Ventana Medical Systems), and sections were blocked for $30 \mathrm{~min}$ with background buffer solution (Innovex). Anti-CD3 antibody (Agilent, Cat\# A0452, RRID:AB_2335677, $1.2 \mu \mathrm{g} / \mathrm{mL}$ ) and anti-HER2 (Enzo Life Sciences Cat\#ALX-810-227L001, RRID: AB_11180914, $5 \mu \mathrm{g} / \mathrm{mL}$ ) were applied, and sections were incubated for 5 hours, followed by $60 \mathrm{~min}$ incubation with biotinylated goat anti-rabbit IgG (Vector laboratories, cat\# PK6101) at 1:200 dilution. The detection was performed with DAB detection kit (Ventana Medical Systems) according to manufacturer's instruction. All images were captured from tumor sections using Nikon ECLIPSE Ni-U microscope and NIS-Elements V.4.0 imaging software.

\section{Statistics}

Statistical analyses of tumor growth and in vitro cytokine release were conducted using area under the curves to obtain numerical values that integrated all parts of the growth curve of tumors. Two-tailed Student's t-test was used to determine statistical difference between two sets of data, while one-way analysis of variance with Tukey's post hoc test was used to determine statistical differences among three or more sets of data. All statistical analyses were performed using GraphPad Prism V.8.0 for Windows (GraphPad Software, La Jolla, CA, www. graphpad. com). A $p<0.05$ was considered statistically significant. Asterisks indicate that the experimental $\mathrm{p}$ value is significantly different from the controls at $* \mathrm{p}<0.05 ; * * \mathrm{p}<0.01$; $* * * \mathrm{p}<0.001, * * * * \mathrm{p}<0.0001$.

\section{RESULTS}

\section{Dual antigens targeting strategies using EAT}

We first chose two target antigens GD2 (disialogangliosides) and HER2 (human epidermal growth factor receptor 2) to test the efficacy of dual-antigen targeting strategies including pooled-EATs (co-administering GD2-EATs and HER2-EATs), alternate-EATs (GD2-EATs alternating with HER2-EATs), dual-EATs (T cells simultaneously armed with GD2-BsAb and HER2-BsAb), and TriAb-EATs ( $\mathrm{T}$ cells armed with trispecific antibody (HER2xGD2xCD3 TriAb)) (figure 1A).

First, in vitro tumor cell killing by EATs (figure 1B) was tested at fixed BsAb arming dose $(0.5 \mu \mathrm{g}$ of each $\mathrm{BsAb} / 1 \times 10^{6} \mathrm{~T}$ cells) with increasing $\mathrm{ET}$ ratios against GD2(+) and/or HER2(+) tumor cell lines (online 
A

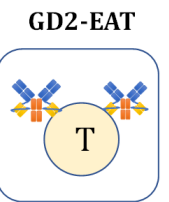

(GD2)

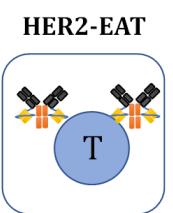

(HER2)

B

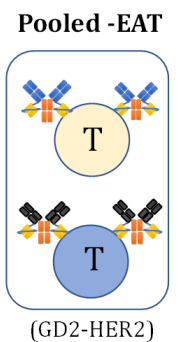

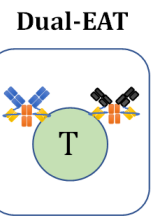

(GD2-HER2)

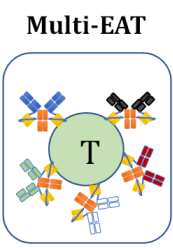

(GD2-HER2 PSMASTEAP1-CD33)
TriAb-EAT

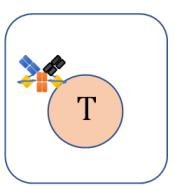

(GD2-HER2)
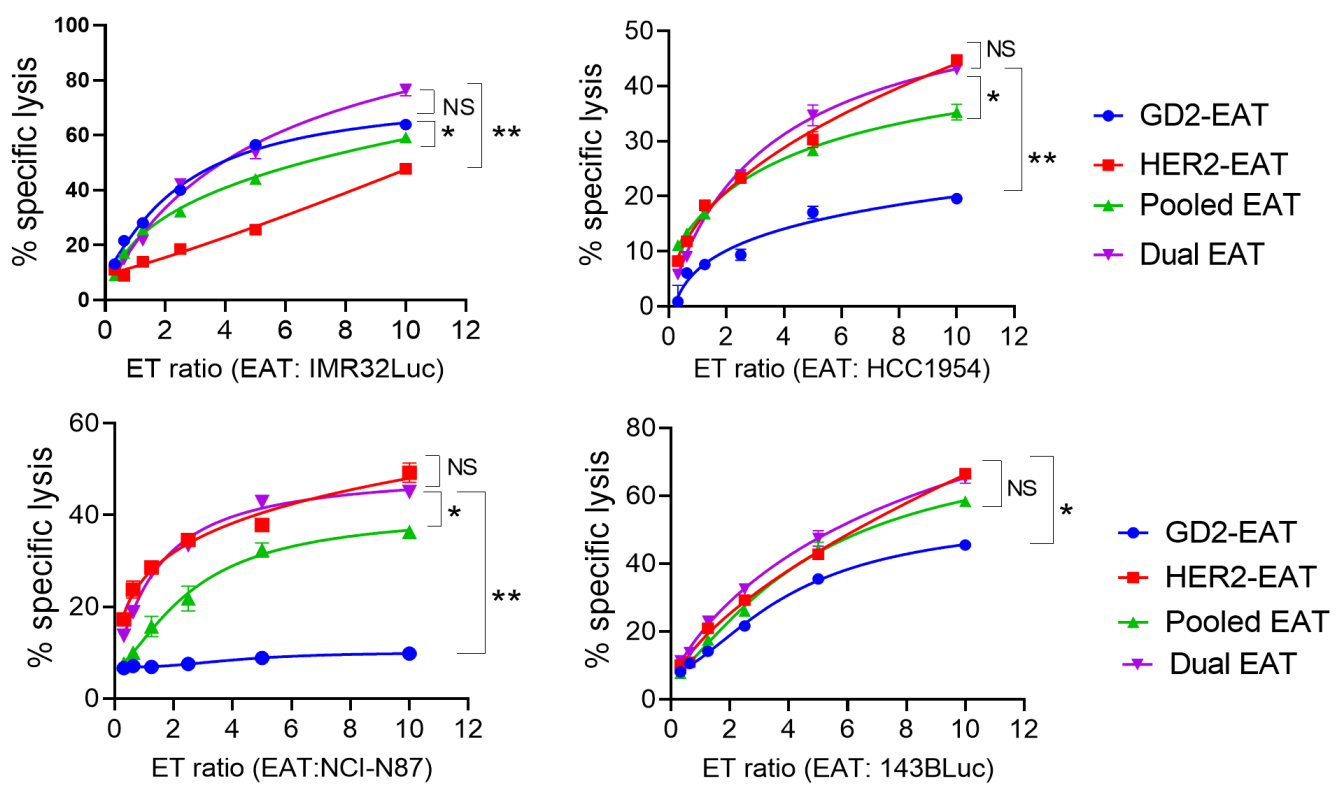

C
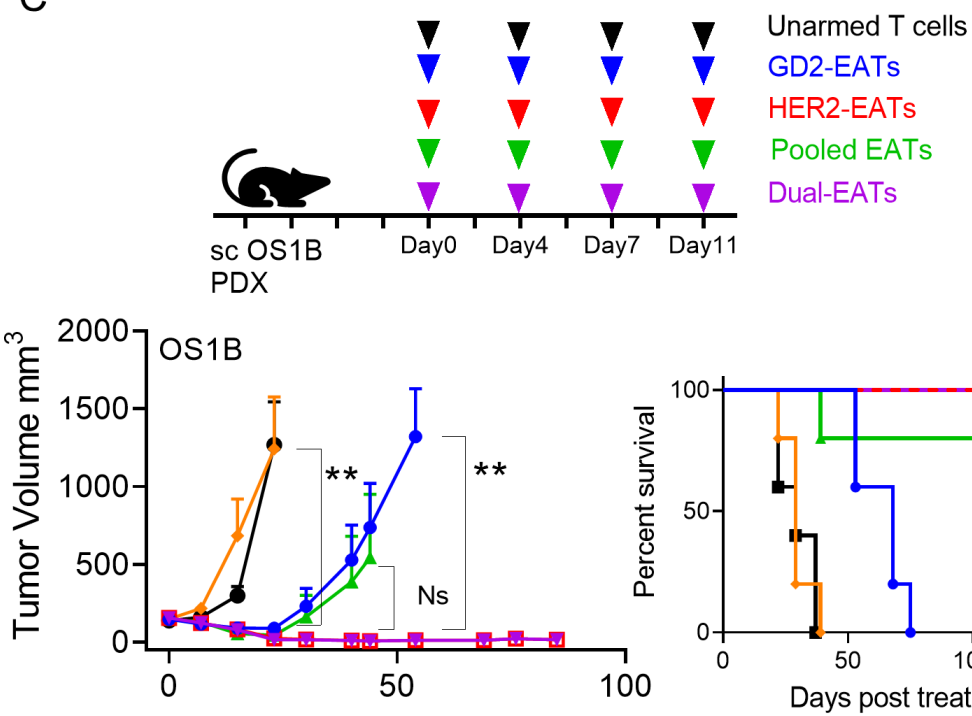

Days post treatment

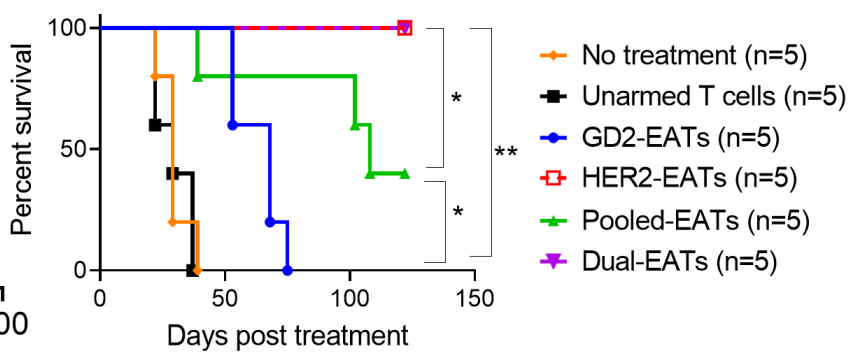

Figure 1 Multiantigens targeting strategies using ex vivo armed I cells with IgG-(L)-scFv platformed BsAb (EATs).

(A) Representative models of mono-EATs (GD2-EATs or HER2-EATs), pooled-EATs, dual- or multi-EATs, and TriAb-EATs, respectively. (B) In vitro cytotoxicity against GD2(+) and/or HER2(+) cancer cell lines was tested and compared among monoEATs, pooled-EATs, and dual-EATs at increasing E:T ratios (effector to target ratio). EATs were armed with $0.5 \mu \mathrm{g}$ of each BsAb per $1 \times 10^{6}$ of T cells. GD2(+) IMR32Luc neuroblastoma cell line, HER2(+) HCC1954 breast cancer cell line, HER2(+) NCl-N87 gastric cancer cell line, and both GD2 and HER2 weakly positive $\left(\mathrm{GD} 2^{10} \mathrm{HER} 2^{10}\right)$ 143BLuc osteosarcoma cell lines were used, respectively. (C) In vivo antitumor response of mono-EATs (GD2-EATs ( $10 \mu \mathrm{g}$ of GD2-BsAb/2 $\times 10^{7}$ cells) or HER2-EATs $(10 \mu \mathrm{g}$ of HER2-BsAb/2 $\times 10^{7}$ cells)), pooled-EATs $\left(5 \mu \mathrm{g} / 1 \times 10^{7}\right.$ of GD2-EATs plus $5 \mu \mathrm{g} / 1 \times 10^{7}$ of HER2-EATs), and dual-EATs $(5 \mu \mathrm{g}$ of GD2$\mathrm{BsAb}+5 \mu \mathrm{g}$ of HER2-BsAb/2 $\times 10^{7}$ cells) was tested against GD2(+) and HER2(+) osteosarcoma PDX (OS1B). Tumor growth curves and overall survival were compared among groups. ${ }^{*} \mathrm{P}<0.05$; ${ }^{* \star} \mathrm{P}<0.01 ;{ }^{* \star \star} \mathrm{P}<0.001 ;{ }^{* \star \star \star} \mathrm{P}<0.0001$. BsAb, bispecific antibody; NS, not significant; PDX, patient-derived xenografted. 
supplemental table S2). Pooled-EATs and dual-EATs showed comparable tumor cell killing when compared with mono-EATs (GD2-EATs or HER2-EATs). While pooled-EATs presented an intermediate potency and efficacy between two mono-EATs, dual-EATs showed a similar potency to the target-specific mono-EATs.

In vivo antitumor effect of multi-EATs was also evaluated using GD2(+) and HER2(+) osteosarcoma PDXs and compared with pooled-EATs (figure 1C). While pooledEATs showed an intermediate anti-tumor effect between two mono-EATs, dual-EATs were equally effective as HER2EATs; all 5 mice in the dual-EATs or HER2-EATs remained progression-free during follow-up period (up to 150 days post-treatment), while none in the GD2-EATs group and only two of five in the pooled-EATs group showed a longterm remission. We also compared in vivo potency of dualEATs with alternate-EATs using an osteosarcoma 143BLuc CDX mouse model (online supplemental figure S1A). The dual-EATs significantly suppressed tumor growth and showed a comparable antitumor effect to HER2-EATs or alternate-EATs without increasing toxicities (online supplemental figure S1B).

Next, the antitumor efficacy of dual-EATs was compared with TriAb-EATs. We developed a novel GD2xHER2xCD3 trispecific antibody (TriAb) built on the $\operatorname{IgG}-(\mathrm{L})-\mathrm{scFv}$ platform using a heterodimeric approach as previously described (figure 2A) ${ }^{15}$ HER2xGD2xCD3 TriAb should engage GD2 and HER2 antigens on tumor cells simultaneously. Their cytotoxicity against multiple cancer cell lines was tested in vitro at fixed BsAb arming dose $\left(0.5 \mu \mathrm{g}\right.$ of each $\mathrm{BsAb} / 1 \times 10^{6} \mathrm{~T}$ cells $)$ with increasing ET ratios (figure $2 \mathrm{~B}$ ). TriAb-EATs were comparable or more effective than GD2-EATs against GD2(+) target cells but were less potent than HER2-EATs against HER2(+) target cells. On the other hand, dual-EATs showed consistently potent cytotoxicity against either GD2(+) and/or HER2 (+) cancer cell lines. In vivo anti-tumor efficacy of TriAb-EATs was tested using two different osteosarcoma PDX models. Three doses of TriAb-EATs successfully ablated PDX tumors, prolonging survival without obvious toxicity in TEOSC1 PDX model (figure 2C). HGSOC1 PDX was more sensitive to GD2-EATs than HER2-EATs, and TriAb-EAT potency was comparable to that of GD2EATs (online supplemental figure S2).

\section{Optimizing BsAb densities on multi-EATs}

Going beyond dual specificities, T cells were simultaneously armed with multiple BsAbs specific for GD2, HER2, CD33, STEAP-1, or PSMA, all built on the IgG(L) -scFv platform. Given the finite CD3 density on human T cells, ${ }^{24}$ we set out to identify the range and the optimal BsAb surface density as a function of arming dose. Surface BsAb density on EAT was analyzed using anti-human IgG Fc-specific antibody. Quantification of $\mathrm{BsAb}$ was measured as $\mathrm{ABC}$ by flow cytometry referenced to quantum beads (figure $3 \mathrm{~A}$ ). As the number of $\mathrm{BsAb}$ and their arming doses increased, BsAb surface density also increased. Arming with 5 BsAbs at high arming dose
( $25 \mu \mathrm{g}$ of each $\mathrm{BsAb} / 10^{6}$ cells), surface density of BsAb plateaued at approximately 50,000 molecules per T cell.

To identify the range of optimal surface density of BsAb for multi-EATs, in vitro cytotoxicity against CD33(+) leukemia cell line (MOLM13) was studied over a range of ET ratios and BsAb arming doses (figure 3B). MultiEATs (armed with 5 BsAbs each targeting GD2, HER2, CD33, STEAP-1, and PSMA, respectively) showed the best cytotoxicity at the arming dose for each BsAb between $0.05 \mu \mathrm{g} / 1 \times 10^{6} \mathrm{~T}$ cells and $0.5 \mu \mathrm{g} / 1 \times 10^{6} \mathrm{~T}$ cells, corresponding to a total BsAb density between 5000 and 20,000 molecules per $\mathrm{T}$ cell.

In vitro antitumor activity of multi-EATs targeting five antigens (GD2, HER2, CD33, PSMA, and STEAP1) was evaluated against varieties of cancer cell lines expressing the respective cognate tumor targets (online supplemental table S2) and compared with mono-EATs over a range of BsAb arming doses (figure 3C). Multi-EATs exerted consistently potent anti-tumor activities against each tumor cell line, comparable to those of mono-EATs, although the maximal cytotoxicity (Emax) was not always comparable.

\section{Ex vivo arming of $T$ cells attenuated cytokine surge from multiple BsAbs}

The simultaneous administration of multiple BsAbs could precipitate a cytokine storm. Cytokine release was compared between multi-EATs and unarmed T cells mixed with multiple BsAbs at increasing doses of BsAb in the presence of tumor targets (figure 4A). Cytokine release from $\mathrm{T}$ cells was BsAb dose dependent, plateauing at $1 \mu \mathrm{g}$ of each $\mathrm{BsAb} / 1 \times 10^{6}$ cells. On the other hand, the cytokine levels of multi-EATs confronted with tumor cells were significantly lower. When we compared the levels of cytokines released by mono-EATs (HER2-EATs), dualEATs (HER2/GD2-EATs), triple-EATs (HER2/GD2/ CD33-EATs), quadruple-EATs (HER2/GD2/CD33/ PSMA-EATs), and quintuple-EATs (HER2/GD2/CD33/ PSMA/STEAP1-EATs), the differences were not significant among groups (figure 4B). Although IL-2, IL-10, IFN- $\gamma$, and TNF- $\alpha$ levels increased with BsAb arming dose, there was no excessive cytokine release when multiple BsAbs were used for arming.

In vivo cytokine levels by multi-EATs were also analyzed post-treatment and compared among groups (figure $4 \mathrm{C}$ ). Multi-EATs ( $50 \mu \mathrm{g}$ of total $\mathrm{BsAb} / 2 \times 10^{7}$ cells, G2) released significantly less IL-2, IL-6, IFN- $\gamma$, and TNF- $\alpha$ than unarmed T cells $\left(2 \times 10^{7}\right.$ cells $)$ with directly injected GD2$\mathrm{BsAb}(10 \mu \mathrm{g})(\mathrm{G} 1)$, and there was no significant difference in cytokine release among the EAT groups: GD2-EATs (G3), HER2-EATs (G4), and multi-EATs (G2).

\section{Multi-EATs as multispecific cytotoxic T lymphocytes}

In vivo antitumor properties against diverse cancer types

In vivo antitumor effect of multi-EATs was tested against multiple cancer xenografts representing different tumor types (figure $5 \mathrm{~A})$. Multi-EATs $(2 \mu \mathrm{g}$ of each $\mathrm{BsAb} \times 5$ BsAbs $/ 2 \times 10^{7} \mathrm{~T}$ cells per injection) significantly suppressed 
(A)

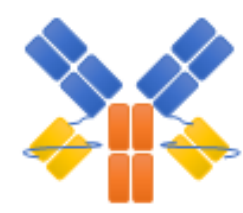

Bivalent GD2

Bivalent CD3 $2+2$ GD2xCD3

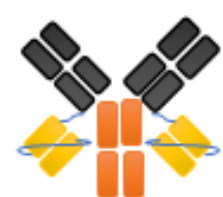

Bivalent HER2

Bivalent CD3 $2+2$

HER2xCD3

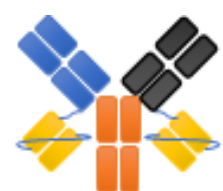

Monovalent GD2

Monovalent HER2

Bivalent CD3

$1+1+2$

GD2xHER2xCD3 TriAb

(B)
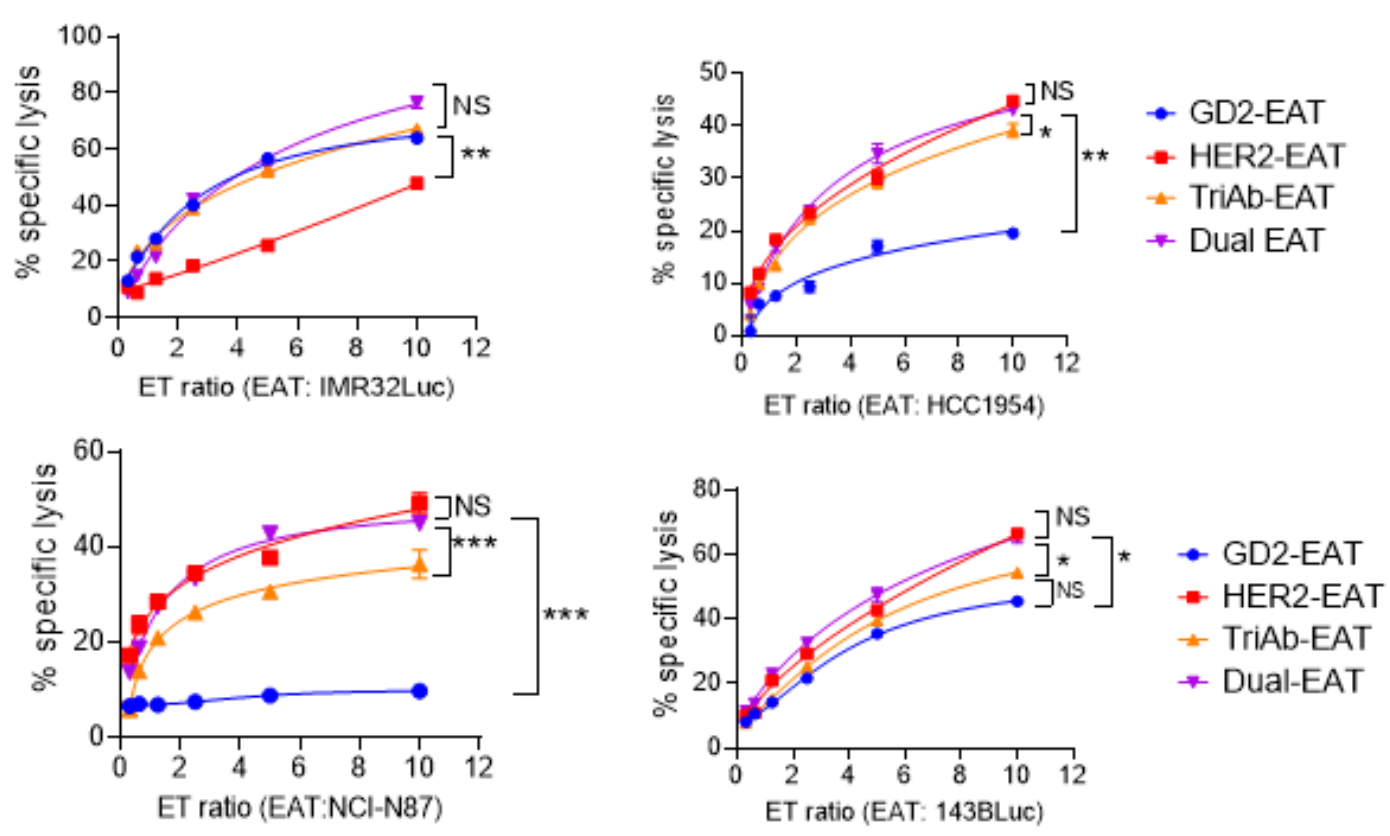

(C)
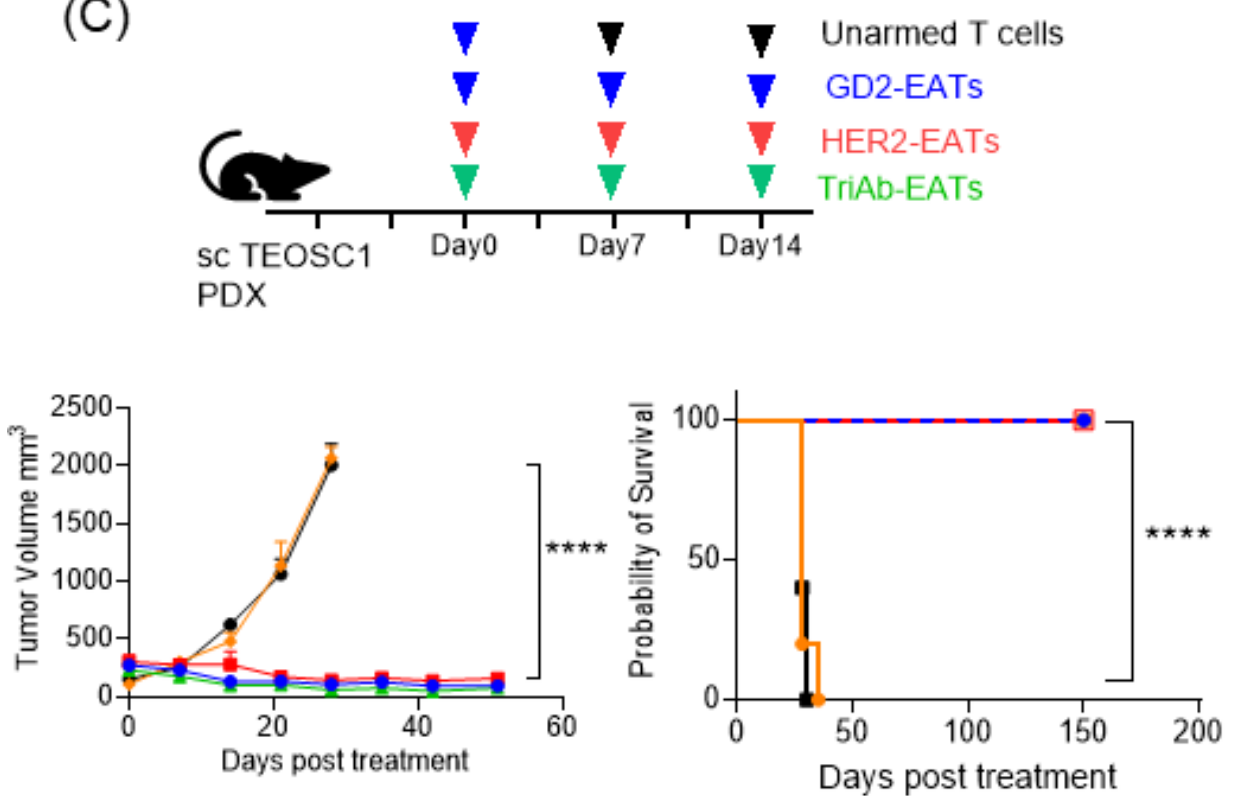

Unarmed T cells $(n=5)$

- No treatment $(n=5)$

- GD2-EATs $(n=5)$

$\because$ HER2-EATs $(n=5)$

$\div$ Tri-Ab-EATs $(n=5)$

Figure 2 Antitumor activity of GD2xHER2xCD3 trispecific antibody (TriAb) armed T cells (TriAb-EATs). (A) Bispecific antibody structure of GD2xHER2xCD3 TriAb. (B) Antibody-dependent T cell-mediated cytotoxicity (ADTC) of TriAb-EAT was compared with mono-EAT (GD2-EAT or HER2-EAT) and dual-EAT against GD2(+) and/or HER2(+) cancer cell lines at increasing E:T ratios. (C) In vivo antitumor effect of TriAb-EATs against GD2(+) and HER2(+) osteosarcoma PDX (TEOSC1). Three doses of unarmed T cells $\left(2 \times 10^{7}\right.$ cells) or EATs $\left(10 \mu \mathrm{g}\right.$ of each $\mathrm{BsAb} / 2 \times 10^{7}$ cells) were administered. ${ }^{*} \mathrm{P}<0.05 ;{ }^{* \star} \mathrm{P}<0.01$; ${ }^{* \star *} \mathrm{P}<0.001 ;{ }^{* \star \star} \mathrm{P}<0.0001$. BsAb, bispecific antibody; E:T, effector to target; NS, not significant; PDX, patient-derived xenografted. 
A

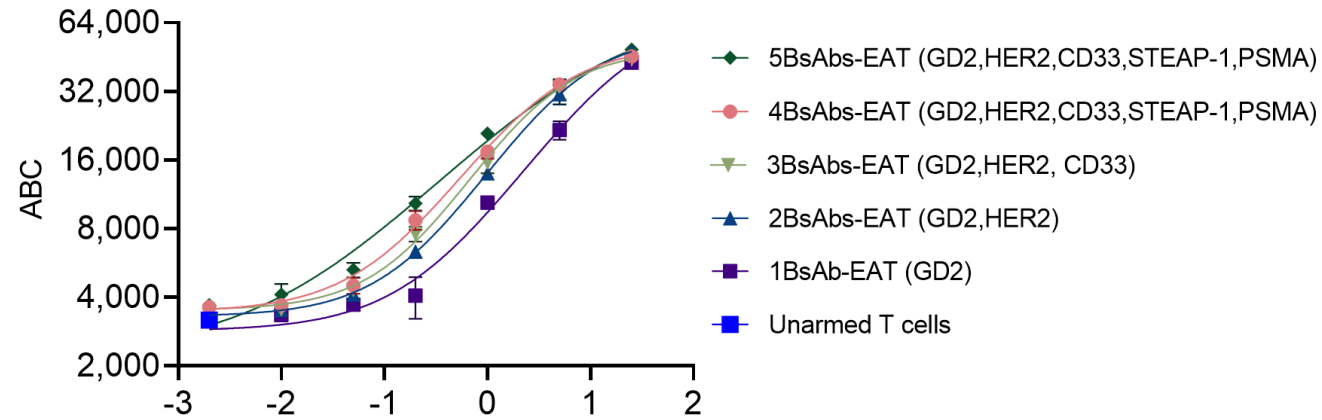

B

Log Each BsAb [Conc], $\mu \mathrm{g} / 1 \times 10^{6} \mathrm{~T}$ cells
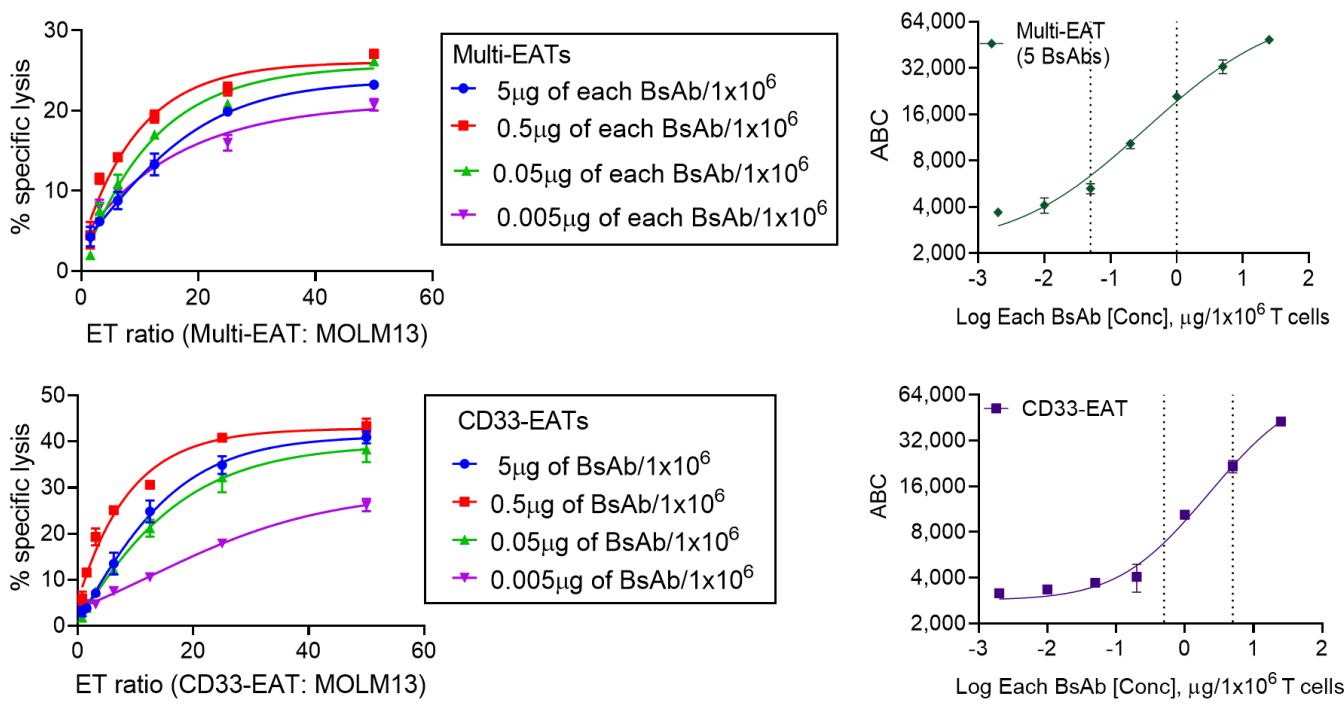

C
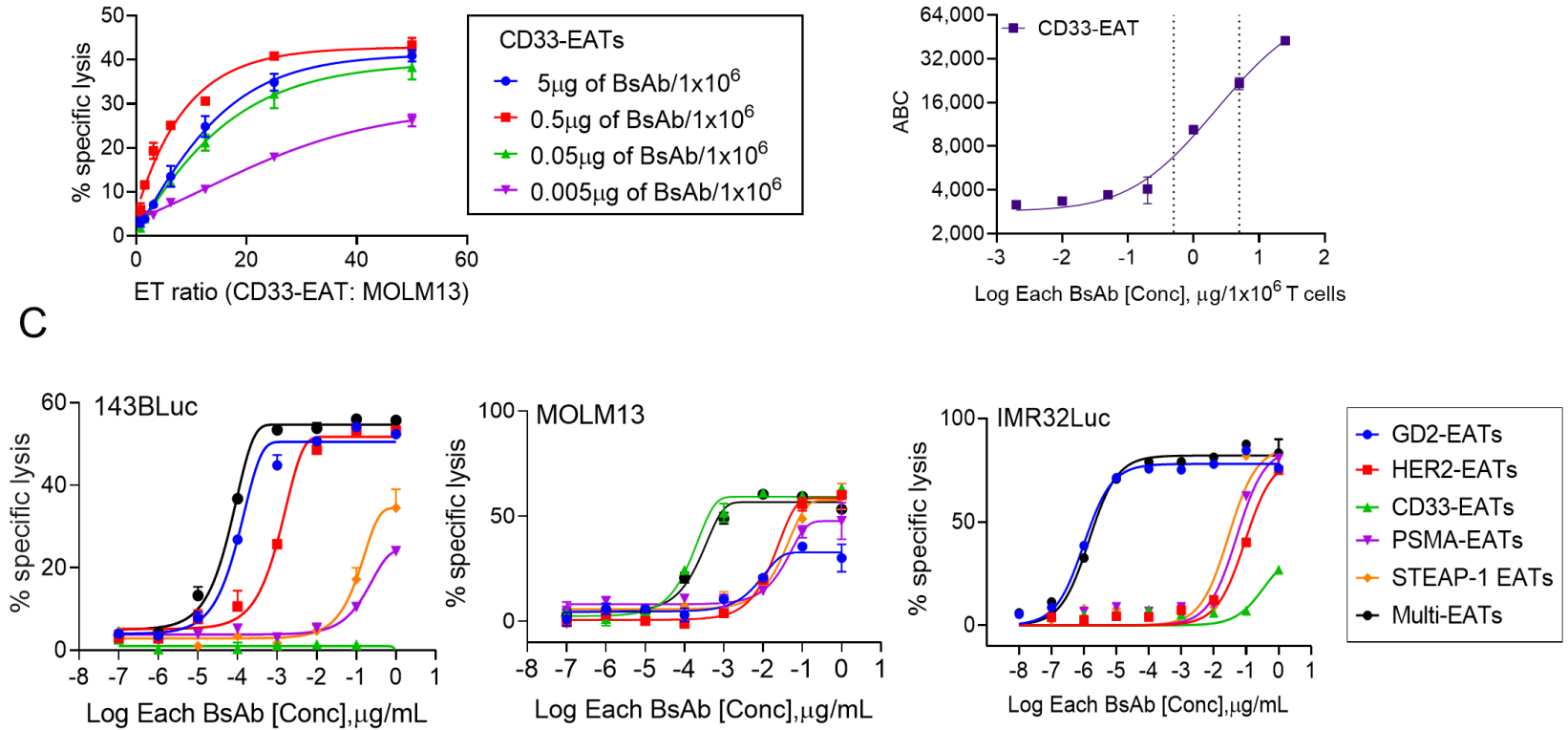

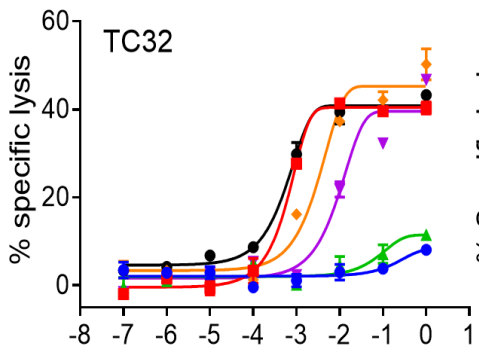

Log Each BsAb[Conc],ug/mL

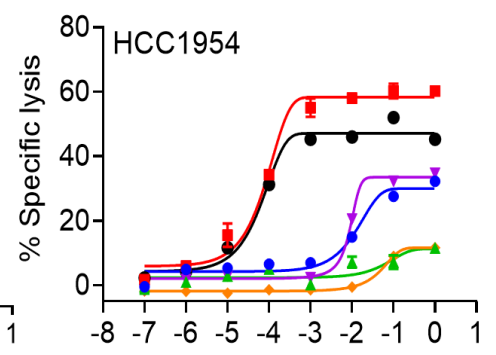

Log Each BsAb [Conc], $\mu \mathrm{g} / \mathrm{mL}$

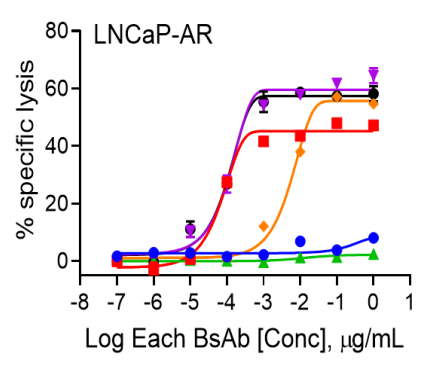

Figure 3 Ex vivo armed T cells with multiple BsAbs (multi-EATs). (A) Surface BsAb density on multi-EAT was analyzed using anti-human IgG Fc-specific antibody and quantified using quantum beads. Geometric mean fluorescence intensities (MFIs) of EATs were measured with increasing arming doses of each BsAb, and BsAb density (MFI) of EAT was referenced to antibodybinding capacity (ABC). (B) In vitro cytotoxicity of multi-EATs and CD33-EATs against CD33(+) MOLM13 cell line at increasing $\mathrm{E}: \mathrm{T}$ ratios and increasing BsAb arming doses. The optimal BsAb densities on T cells were extrapolated from the ADTC assays. (C) In vitro cytotoxicity of multi-EATs was tested against a panel of tumor cell lines (E:T ratio was 10:1) and compared with mono-EATs. ABC, antibody binding capacity; ADTC, antibody-dependent T cell-mediated cytotoxicity; BsAbs, bispecific antibodies; EATs, ex vivo armed T cells; E:T, effector to target. 
A
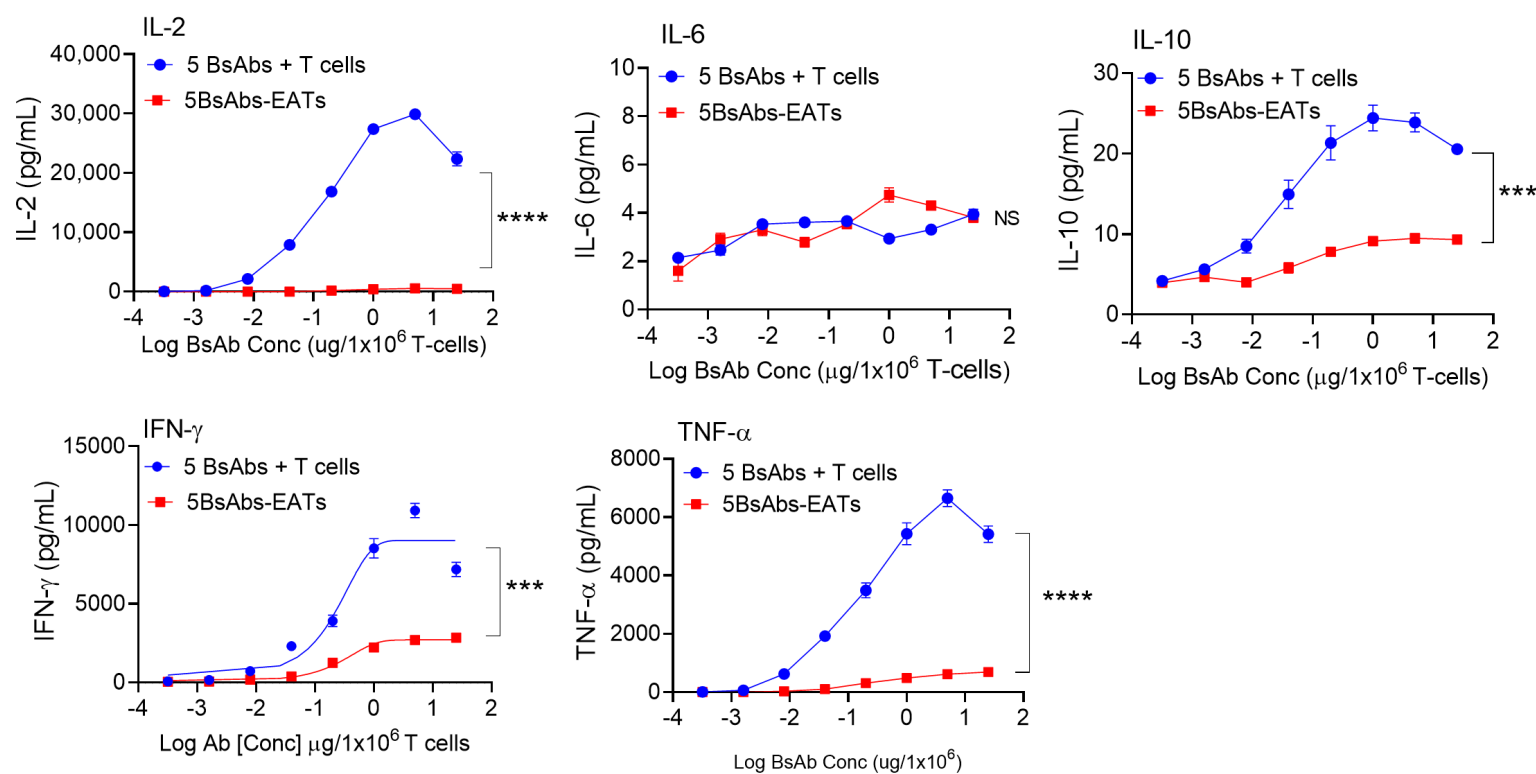

B
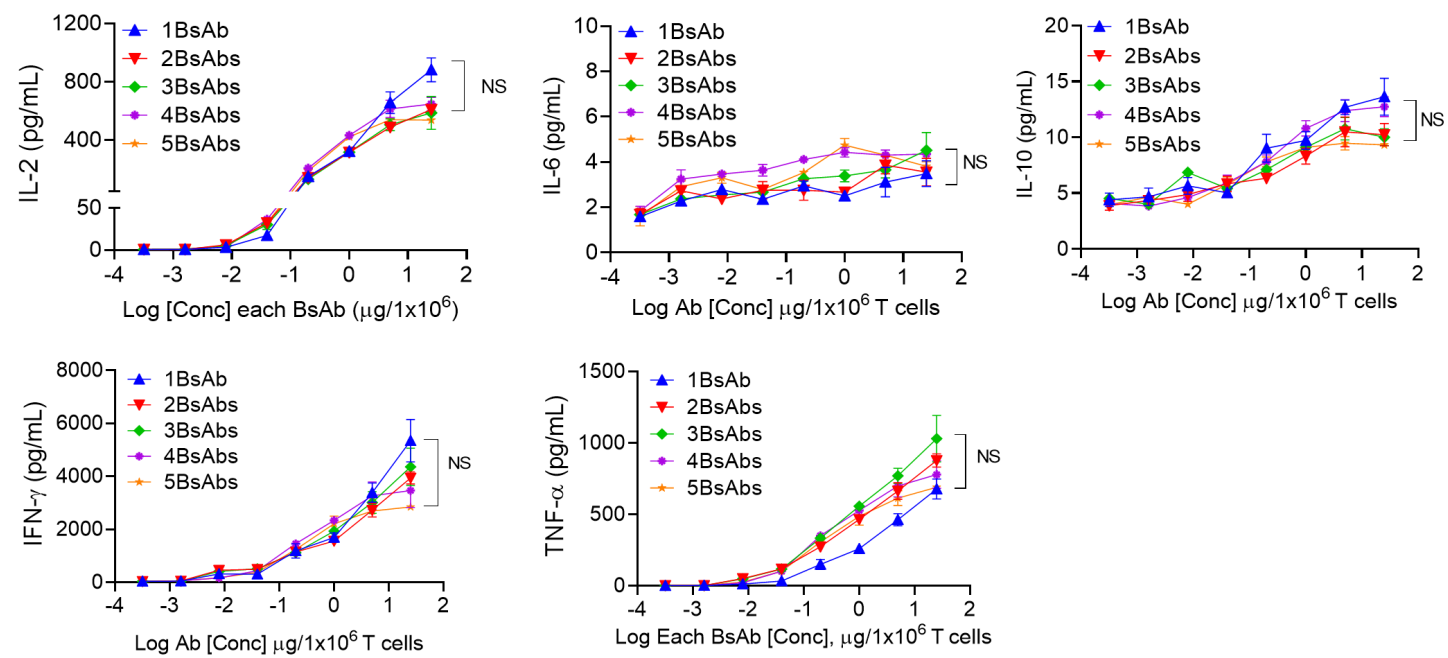

C
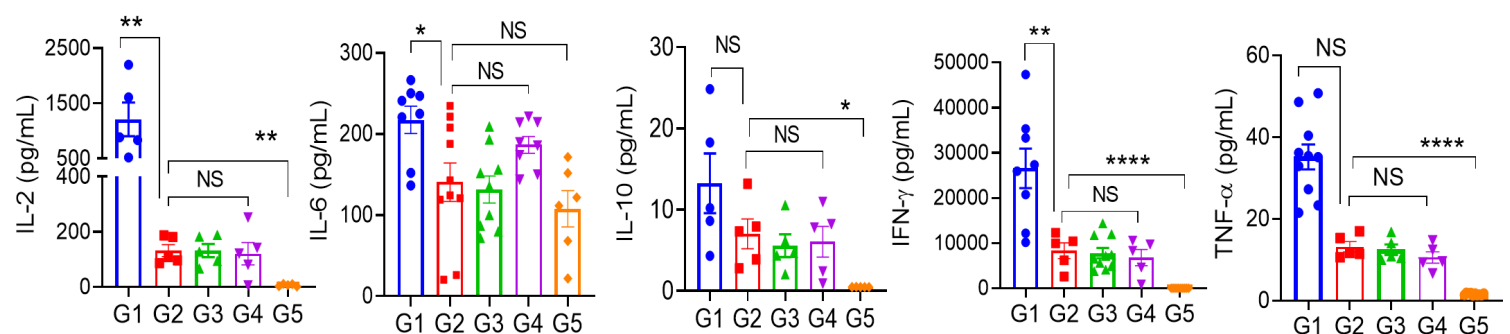

Figure 4 Cytokine release by multiple BsAbs plus T cells and multi-EATs. (A) TH1 cell cytokines (IL-2, IL-6, IL-10, IFN- $\gamma$, and TNF- $\alpha$ ) were measured in the supernatants after 4 hours of incubation of 5 BsAbs plus T cells or 5 BsAb armed T cells (5BsAbsEATs) with target cells at increasing doses of each BsAb $\left(0.0003 \mu \mathrm{g} / 1 \times 10^{6}\right.$ cells to $25 \mu \mathrm{g} / 1 \times 10^{6}$ cells). Mixture of multiple cancer cell lines consisting GD2(+) M14Luc, HER2(+) HCC1954, CD33(+) HL60, PSMA(+) LNCaP-AR, and STEAP1(+) TC32 were used as target cells. ET ratio (effector to target cell ratio) was 20:1. (B) In vitro cytokine release of multi-EATs was compared following an increase in the number of BsAb. ET ratio was 20:1, and mixture of multiple cancer cell lines consisting GD2(+) M14Luc, HER2(+) HCC1954, CD33(+) HL60, PSMA(+) LNCaP-AR, and STEAP1(+) TC32 were used as target cells. (C) In vivo TH1 cytokine levels were analyzed 4 hours after second dose of EAT in GD2 ${ }^{10} \mathrm{HER} 2^{\text {lo }} 143 \mathrm{BLuc}$ osteosarcoma cell line xenograft (CDX) mouse model. G1, GD2-BsAb and unarmed T cells; G2, multi-EATs (GD2/HER2/CD33/PSMA/STEAP1-EATs); G3, GD2-EATs; G4, HER2-EATs; G5, unarmed T cells. BsAb dose and T cell number were fixed at $10 \mu \mathrm{g}$ for each BsAb and $2 \times 10^{7}$ for T cell per injection. ${ }^{*} \mathrm{P}<0.05 ;{ }^{* *} \mathrm{P}<0.01 ;{ }^{* \star *} \mathrm{P}<0.001 ;{ }^{* \star \star *} \mathrm{P}<0.0001$. BsAbs, bispecific antibodies; EATs, ex vivo armed $\mathrm{T}$ cells; $\mathrm{ET}$, effector to target; NS, not significant. 

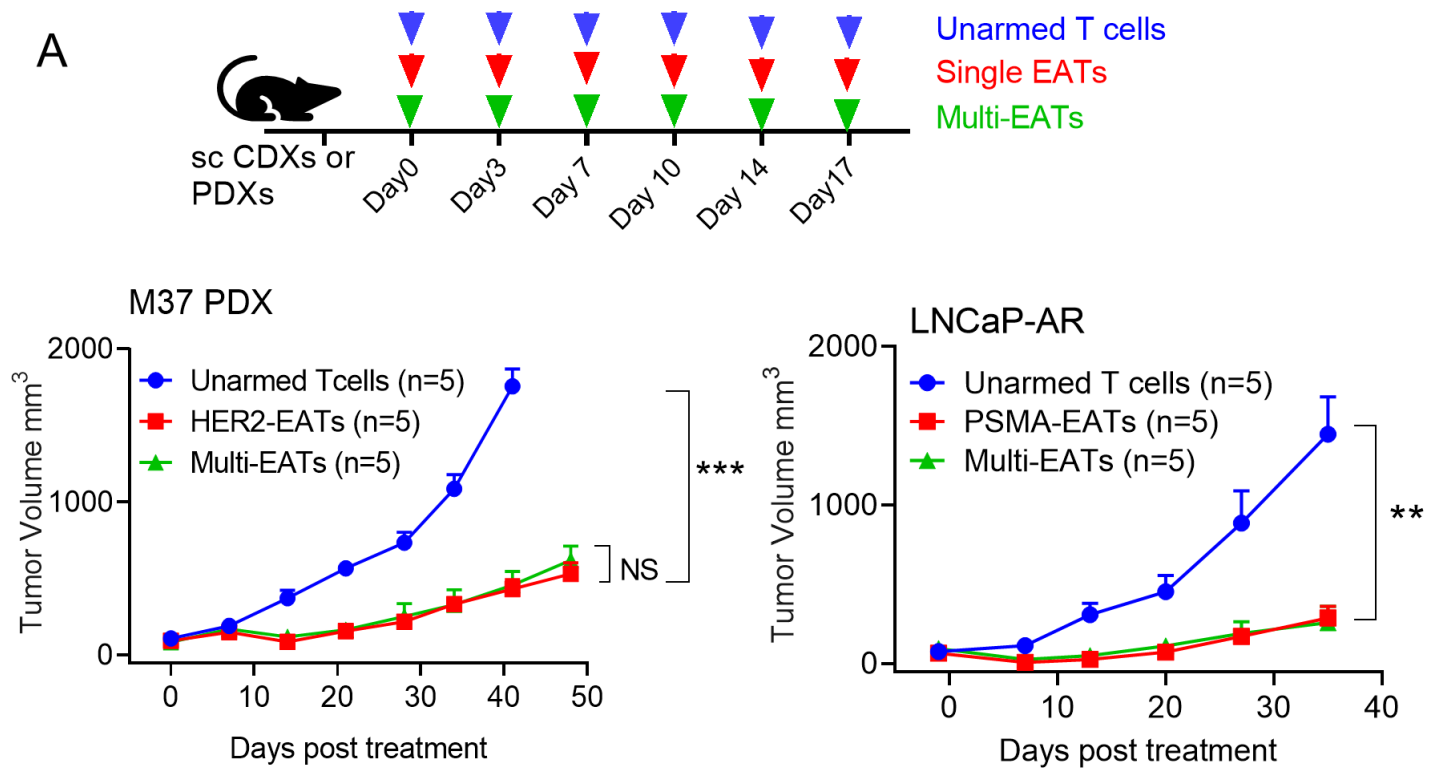

IMR32LuC
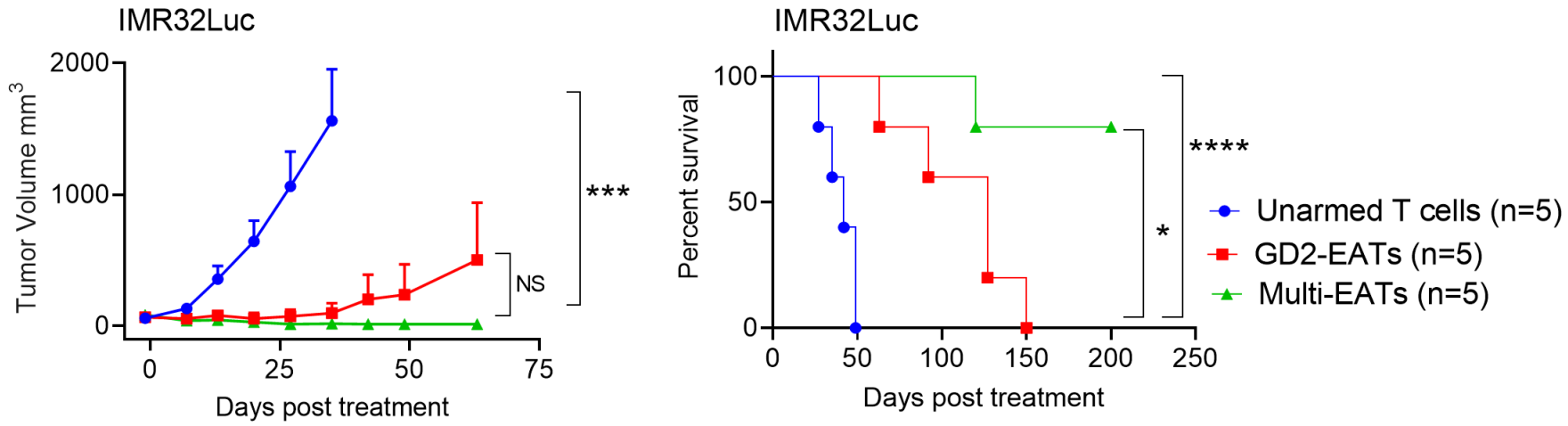

B
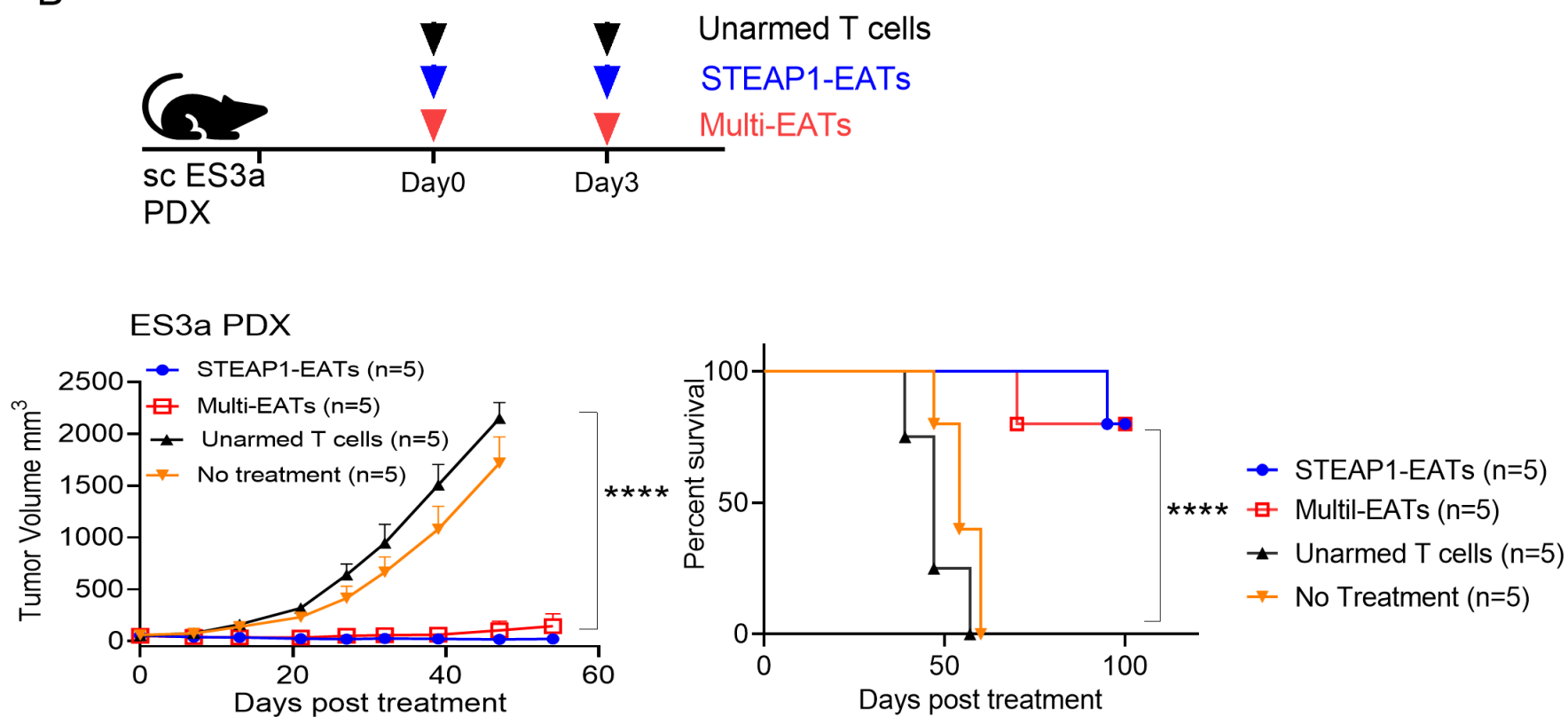

Figure 5 In vivo antitumor activities of multi-EATs. (A) In vivo antitumor effect of multi-EATs was tested against a variety of cancer xenografts including M37 breast cancer PDXs, LNCaP-AR prostate cancer CDXs, and IMR32Luc neuroblastoma CDXs. Six does EATs or unarmed T cells were administered. (B) In vivo antitumor effect of multi-EATs was compared with single antigen targeted STEAP1-EATs against Ewing sarcoma family of tumor (EFT) PDXs. Two doses of EATs or unarmed T cells were administered. BsAb dose and T cell number were fixed at $2 \mu \mathrm{g}$ for each $\mathrm{BsAb}$ and $2 \times 10^{7}$ for $\mathrm{T}$ cell per injection. ${ }^{*} \mathrm{P}<0.05$; ${ }^{* \star} \mathrm{P}<0.01 ;{ }^{* \star *} \mathrm{P}<0.001 ;{ }^{* \star \star *} \mathrm{P}<0.0001$. EATs, ex vivo armed T cells; BsAb, bispecific antibodies; NS, not significant; PDX, patientderived xenografted. 
tumor growth and consistently showed competitive antitumor effects when compared with mono-EATs against a panel of target-positive cancer xenografts, including HER2(+) M37 breast cancer PDX, PSMA(+) LNCaP-AR prostate cancer CDX, GD2(+) IMR32Luc neuroblastoma CDX, and STEAP1(+) ES3a EFT PDXs (figure 5B), without clinical toxicities. For IMR32Luc CDXs, multiEATs exerted a robust antitumor effect surpassing the efficacy of GD2-EATs, significantly prolonging survival.

\section{Multi-EATs were highly effective against tumor models with antigen heterogeneity}

We next studied the potential of multi-EATs to overcome tumor heterogeneity by creating a mixed lineage, that is, GD2(+) IMR32Luc mixed with HER2(+) HCC1954 (1:1 ratio) (figure 6A). Dual-EATs (T cells armed with GD2BsAb and HER2-BsAb) and multi-EATs (T cells armed with 5 BsAbs targeting GD2, HER2, CD33, PSMA, and STEAP1, respectively) induced stronger cytotoxicity than GD2EATs or HER2-EATs against these mixed lineage cancer cells (figure 6B). This enhanced in vitro cytotoxicity of dual-EATs or multi-EATs translated into more potent in vivo anti-tumor response. The mixed lineage cancer cells were xenografted subcutaneously and treated with EATs armed at $10 \mu \mathrm{g}$ of each $\mathrm{BsAb} / 2 \times 10^{7} \mathrm{~T}$ cells (figure $6 \mathrm{C}$ ). No clinical toxicities were observed, and there was no weight loss throughout the follow-up period (figure 6D). While GD2-EATs or HER2-EATs failed to produce durable responses against this mixed lineage CDX, dual-EATs, multi-EATs, or alternate-EATs successfully induced tumor regression, producing long-term survival (figure $6 \mathrm{E}, \mathrm{F}$ ). Dual-EATs or multi-EATs both surpassed the efficacy of mono-EATs, significantly improving tumor-free survival (vs HER2-EATs, $\mathrm{p}=0.0033$; vs GD2-EATs, $\mathrm{p}<0.0001$ ). There was no significant difference in antitumor efficacy among dual-EATs, multi-EATs, and alternate-EATs.

We also tested the efficacy of TriAb-EATs against this mixed lineage. While TriAb-EATs showed enhanced in vitro cytotoxicity compared with GD2-EATs or HER2EATs, it was not as effective when compared with dualEATs or multi-EATs (online supplemental figure S3A). In vivo anti-tumor activity of TriAb-EATs was also tested against this mixed lineage CDXs (online supplemental figure S3B). Tumors regressed following TriAb-EATs, but the response was not durable: all 5 mice relapsed after short-term response, contrasting with groups treated with dual-EATs or multi-EATs where long-term disease-free survival extended past 140 days in three out of five and four out of five mice, respectively.

\section{Multi-EATs overcame tumorous heterogeneity: histological response of mixed lineage CDX to multi-EATs}

The IMR32Luc and HCC1954 mixed lineage CDXs were harvested and analyzed their antigen expression (online supplemental table S3). Gross examination of these tumors presented distinct differences between IMR32Luc and HCC1954 lineages (figure 7A and online supplemental figure S4A). Following treatment with GD2-EATs or TriAb-EATs tumors grossly resembled HCC1954 CDXs, while following HER2-EATs relapsed tumors appeared IMR32Luc CDXs. Recurrent tumors following alternateEATs, dual-EATs, or multi-EATs had the appearances of IMR32Luc intermixed with HCC1954 CDXs. On the other hand, untreated tumors or those treated with unarmed T cells more resembled HCC1954 CDXs, consistent with rapid outgrowth of HCC1954 overtaking IMR32Luc. The gross pathologic phenotypes were confirmed by H\&E staining (figure 7B and online supplemental figure $\mathrm{S} 4 \mathrm{~B}$ ). While following treatment with GD2-EATs or TriAb-EATs histology revealed poorly-differentiated invasive ductal breast carcinoma, following treatment with HER2-EATs tumor histology revealed immature, undifferentiated, small round neuroblasts accompanied by Homer-Wright pseudorosettes, typical characteristics of neuroblastoma. In the groups receiving no treatment or unarmed $\mathrm{T}$ cells, or in groups with recurrence after initial response to alternate-EATs, dual-EATs or multi-EATs, the histology revealed a mixed lineage with a slight prominence of breast cancer features. Fresh frozen tumor staining with anti-GD2 antibody (hu3F8) (figure 7C and online supplemental figure S4C) and FFPE tumor staining with anti-human HER2 antibody (figure 7D and online supplemental figure S7D) also showed corresponding disappearance of antigen with tumor response. While the tumors receiving no treatment or unarmed $\mathrm{T}$ cells showed heterogeneous staining patterns, those treated with GD2EATs or TriAb-EATs became GD2 negative and HER2 strongly positive; vice versa, those tumors treated with HER2-EATs were strongly GD2 positive and HER2 negative. Mono-EATs successfully ablated target antigen positive tumor cells but did not affect target antigen negative clones. On the other hand, the escaped tumors following treatment with dual-EATs, alternate-EATs, or multi-EATs were both GD2 and HER2 weakly positive. Total target antigen loss seen with mono-EATs was not observed in tumors treated with these multiantigen targeting EATs, since the recurrent tumors responded well to retreatment with multi-EATs (online supplemental figure S5).

\section{DISCUSSION}

To test the hypothesis that $\mathrm{T}$ cells with multiple synthetic specificities could overcome tumor heterogeneity preventing clonal escape and the development of treatment resistance, we explored the potential of multiantigen targeting EAT strategies. Multi-EATs, $\mathrm{T}$ cells armed ex vivo with multiple BsAbs, were more effective than pooling of monospecific EATs (pooled EATs) or multiplying specificities on the same protein molecule (TriAb-EATs). While mono-EATs could ablate tumors in an exquisitely antigen-specific manner and were unable to control antigen negative clones in the mixed lineage tumor system, dual- or multi-EATs showed potential to overcome tumor heterogeneity and target antigen loss, two major limitations of current $\mathrm{T}$ cell immunotherapy. 
A

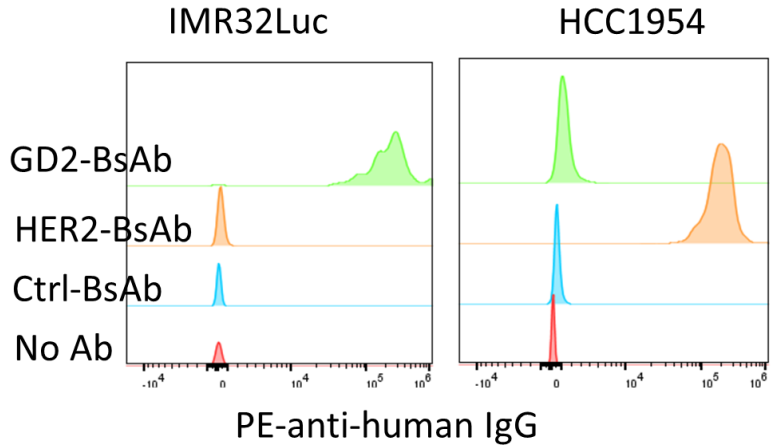

B

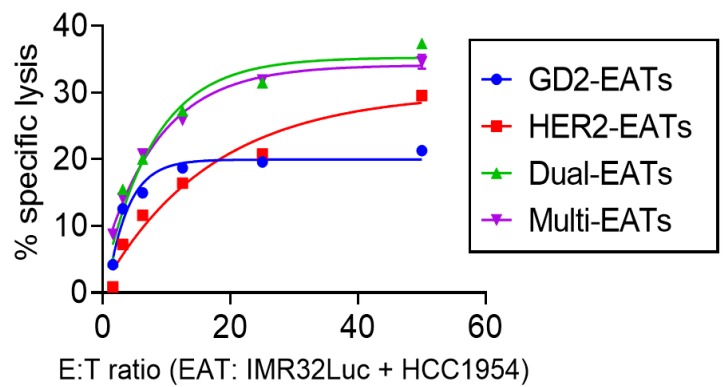

C

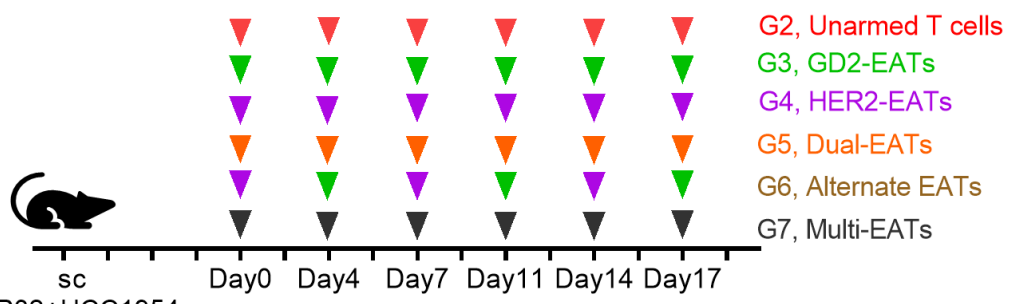

IMR32+HCC1954

D

$E$
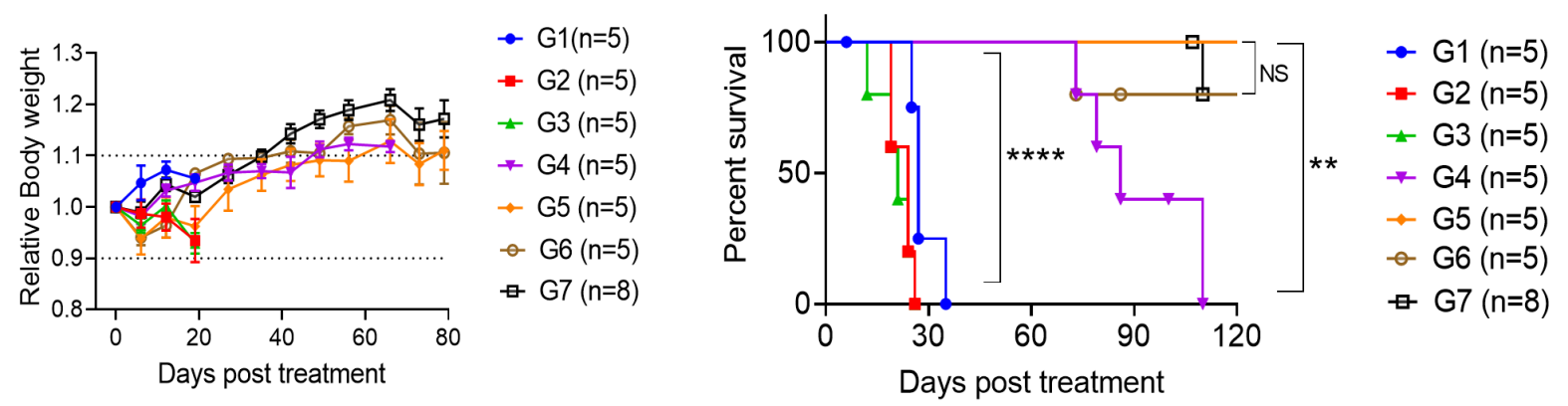

F
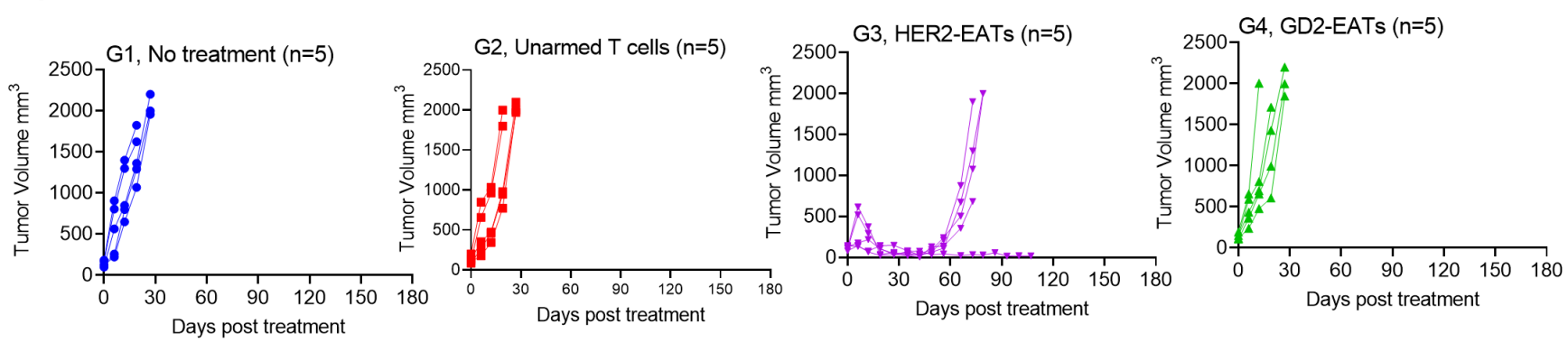

G5, Dual-EATs $(n=5)$
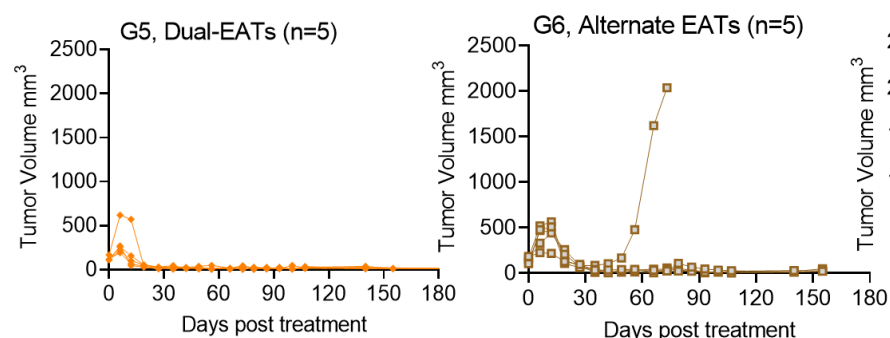

G7, Multi-EAT $(n=8)$

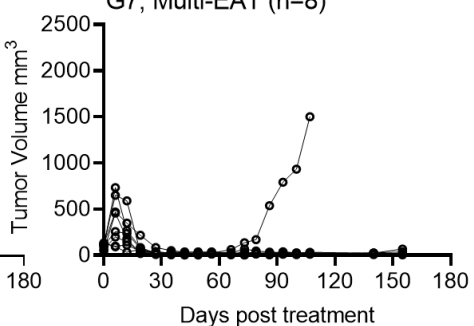

Figure 6 Antitumor efficacy of multi-EATs against mixed lineage targets. (A) Antibody binding intensities (MFls) of each cancer cell line. (B) In vitro cytotoxicity was tested against IMR32Luc and HCC1954 mixed lineage. (C) Schematic overview of treatment for IMR32Luc and HCC1954 mixed lineage xenografts using multi-antigen targeting EAT strategies. BsAb dose and T cell number were fixed at $10 \mu \mathrm{g}$ for each BsAb and $2 \times 10^{7}$ for T cell per injection. (D) Mouse body weight during follow-up period. (E) Overall survival by treatment. (F) Tumor response by treatment groups. ${ }^{\star} \mathrm{P}<0.05 ;{ }^{* \star} \mathrm{P}<0.01 ;{ }^{* \star \star} \mathrm{P}<0.001 ;{ }^{* \star \star \star} \mathrm{P}<0.000$. BsAb, bispecific antibodies; EATs, ex vivo armed T cells; MFIs, mean fluorescence intensities; NS, not significant. 
A

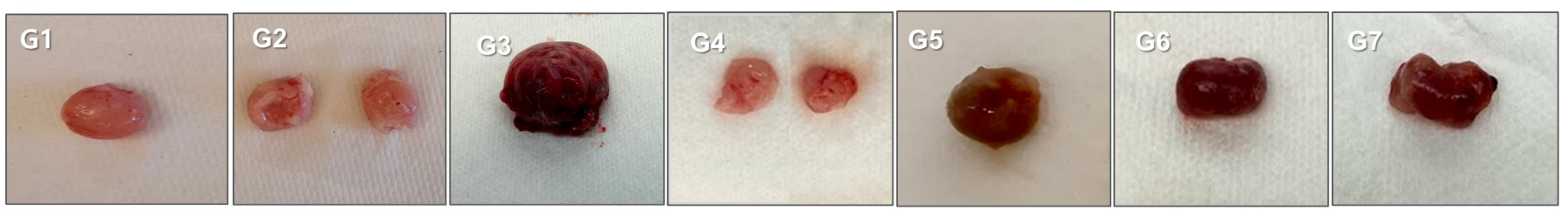

B
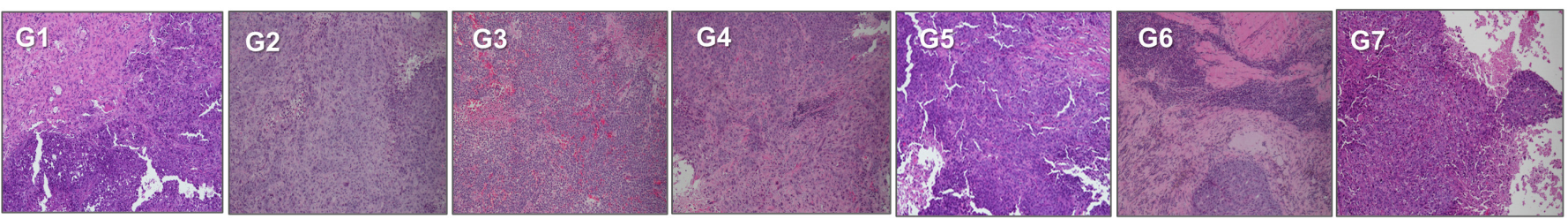

C
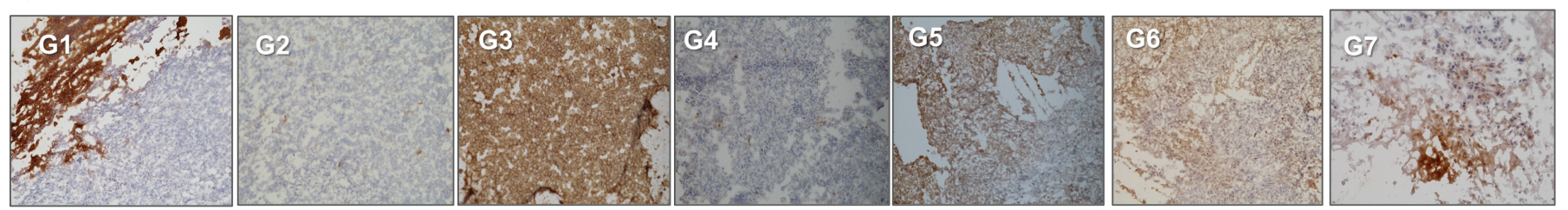

D
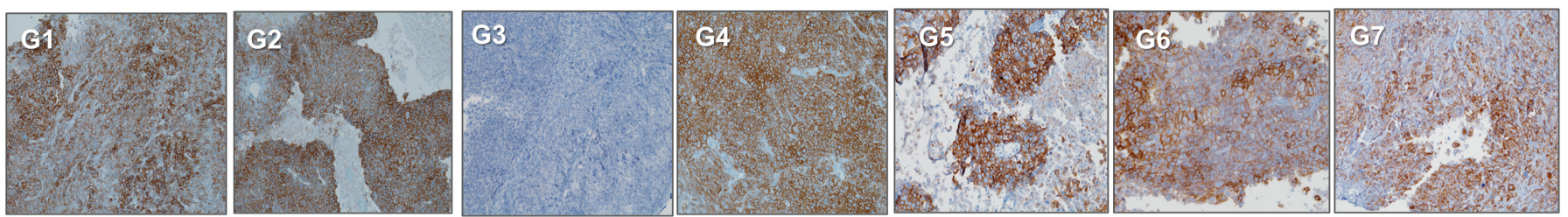

Figure 7 Analysis of tumor response by immunohistochemical (IHC) staining. (A) Gross phenotypes of tumors in each treatment group. (B) H\&E staining of tumors in each treatment group. (C) Fresh frozen tumor staining with anti-human GD2 antibody (hu3F8). (D) IHC staining of formalin-fixed paraffin-embedded (FFPE) tumor sections with anti-human HER2 antibody. (E) IHC staining of FFPE tumor sections with anti-human CD3 antibody. G1, unarmed T cells; G2, GD2-EATs; G3, HER2-EATs; G4, TriAb-EATs; G5, alternate-EATs; G6, dual-EATs; G7, multi-EATs. EATs, ex vivo armed T cells.

Most of human cancers show heterogeneous antigen expression, and single antigen targeted approaches are rarely curative. Furthermore, the TAAs undergo downregulation, mutation, or loss under selective immune pressure following $\mathrm{T}$ cell immunotherapy. ${ }^{25}$ Multi-antigen targeted strategies have the potential to overcome these antigen escape mechanisms. Simultaneous targeting CD19/CD20 or CD19/CD20/CD22 using 'OR' logicgated tandem CAR T cells ${ }^{26}$ reduced or prevented target antigen escape, offering an advantage over single CAR $\mathrm{T}$ cells or pooled populations of monospecific CAR $\mathrm{T}$ cells. ${ }^{27-30}$ Given the minimal requirement of BsAb (only 500-5000 molecules) per T cell for antitumor activity, ${ }^{13}$ multiple BsAbs built on the same IgG-(L)-scFv platform can be installed on each $\mathrm{T}$ cell before the maximum capacity is reached $(30,000-56,000$ molecules per $\mathrm{T}$ cells $\left.{ }^{132}\right)$. Since $\mathrm{T}$ cell loading is mediated through the same anti-CD3 $\mathrm{scFv}$ in IgG-(L)-scFv constructs, BsAb surface density should be predictable and consistent. By adjusting the arming doses, the relative density of each $\mathrm{BsAb}$ on each $\mathrm{T}$ cell could be fine-tuned and optimized. The same Boolean logic for 'OR' and 'AND' gates for CAR T cell ${ }^{2631}$ can be applied to multi-EATs, using appropriately designed BsAbs that can either activate or inhibit
$\mathrm{T}$ cell function. In our studies, dual-EATs or multi-EATs showed a synergistic anti-tumor effect when simultaneously encountering multiple antigens. The formation of bivalent or multivalent immune synapses when dual-EATs or multi-EATs exposed to heterogeneous tumors co-expressing multiple TAAs would be crucial to exert synergistic antitumor effect and prevent antigen escape. ${ }^{28}$

Besides tumor heterogeneity, antigen loss or downregulation has been another challenge to immunotherapy. ${ }^{3}$ CD19 loss or mutation following CD19-CAR T cells, or CD22 density dwindling after CD22-CAR T cell therapy were associated with treatment resistance or relapse. ${ }^{32-35}$ To address this issue, dual antigen targeting strategies, such as CD19/CD22 dual-specific CAR T cells, pooling of CD19- and CD22- CAR T cells, or sequential treatment with CD19- and CD22- CAR T cells, have been explored with variable success. ${ }^{36}$ Our data support an alternative approach using proteins instead of genes to expand T cell specificity. In contrast to the tumors treated with monospecific GD2-EATs or HER2-EATs forcing target antigen loss, the relapsed tumors following alternate-EATs, dualEATs or multi-EATs therapy retained their target antigen expression, and escaped EFT PDXs after multi-EAT therapy responded to re-challenges, implicating a major 
advantage over conventional single antigen-targeted immunotherapy.

One of the concerns of multi-antigen targeted $\mathrm{T}$ cell immunotherapies is 'on-target off-tumor' toxicities. 'On-target off-tumor' toxicities following the infusion of CAR T cells or BsAbs can cause serious or life-threatening adverse effects, and the extent and severity of toxicity could be amplified by increasing numbers of targeting antigens. Although we did not observe any additional toxicity related to multi-EATs, the mouse models we used have major limitations because of the specificity of BsAbs for human not mouse antigens. High target antigen affinity could increase the severity of on-target off-tumor toxicities, while reducing target antigen affinity to a certain threshold $\left(\mathrm{Kd}<10^{-8} \mathrm{M}\right)$ could decrease toxicities without affecting anti-tumor efficiency of T cells. ${ }^{37}$ CAR $\mathrm{T}$ cells with $\mu \mathrm{M}$ affinity retained strong antitumor activity while lowering off-tumor toxicities than their $\mathrm{nM}$ affinity counterparts, ${ }^{38}$ suggesting that avidity optimization is an effective strategy to reduce on-target off-tumor recognition by multivalent targeted immunotherapy. ${ }^{39}{ }^{40}$ MultiEATs take advantage of avidity-affinity balance with the potential to reduce the 'on-target off-tumor' side effects while expanding the spectrum of responsive tumor types. But more importantly, while multi-specific CAR T cells are lifelong and such toxicities could be prolonged and life-threatening, EATs have limited functional life expectancy; as the BsAbs get metabolized, T cells should revert to their nonspecific states, alleviating the risk of lifethreatening long-term toxicities. ${ }^{16}$

Another limitation of the humanized mouse model is the inherent species differences that thwart a faithful mimicking of human diseases and their therapeutics. While the effector $\mathrm{T}$ cells, tumors, and BsAbs were of human origin, the xenograft TME contained cells of mouse origin, including tumor infiltrating myeloid cells, fibroblasts, vasculature, and even lymphatics, which all could interact to influence tumorigenesis and antitumor response. ${ }^{41}{ }^{42}$ A fully syngeneic mouse model in an immunocompetent host has other limitations, including rejection of human BsAbs, use of artificial and not human-derived tumors, and inability to engage human $\mathrm{T}$ cells. ${ }^{41}$ Even though in most studies TIMs have a near-complete congruence between human and mouse, ${ }^{43}$ we remained cautious not to extrapolate lessons from mouse studies to human diseases without clinical validation.

In conclusion, multi-EATs, T cells armed with multiple BsAbs built on the IgG-(L)-scFv platform, retained tumor selectivity and anti-tumor potency both in vitro and in vivo without excessive cytokine release. Multi-EATs demonstrated robust anti-tumor efficacy compared with mono-EATs against individual tumor targets, with the added advantages of being a plug-N-play system offering simplicity in manufacturing. Akin to multi-agent chemotherapy, multi-EATs have the potential to increase potency, to reduce toxicity, and to overcome tumor heterogeneity and clonal escape. This property of multi-EATs deserves further exploration and optimization in order to confront cancer resistance.

Acknowledgements We would like to thank Dr Hong Xu for providing the anti-HER2 BsAb and performing binding kinetic studies for BsAbs, Dr Sayed Shahabuddin Hoseini for anti-CD33 BsAb, Dr Steven Tsung-Yi Lin for the anti-STEAP1 and anti-PSMA BsAb, and Dr Brian H. Santich for creating the GD2xHER2xCD3 trispecific antibody. We want to acknowledge Hong-fen Guo for flow cytometry analysis of tumor cell lines and her expertise in the preparation and biochemical analyses of BsAbs, Yi Feng for staining fresh frozen tumor tissues with hu3F8 antibody, Dr Afsar Barlas for immunohistochemical staining of tumor sections, and Drs Sarah Katz and lleana Miranda in the Laboratory of Comparative Pathology for reviewing pathology of the xenografts. We also thank Dr Elisa De Stanchina for providing PDXs for these studies.

Contributors JAP and N-KVC designed and performed the experiments, interpreted and analyzed the results, and wrote the manuscript. Both two revised this manuscript. All authors had access to the data, reviewed this manuscript, approved final the manuscript, and controlled the decision to publish it. Both authors accept full responsibility for the work and the conduct of the study.

Funding This work was supported by funds to NKC from R01 CA 182526, Enid A. Haupt Endowed Chair, the Robert Steel Foundation, and Kids Walk for Kids with Cancer. Technical service provided by the MSK Animal Imaging Core Facility, Antitumor Assessment Core Facility, and Molecular Cytology Core Facility were supported in part by the NCI Cancer Center Support Grant P30 CA008748.

Competing interests Both N-KVC and JAP were named as inventors on the patent of EATs filed by MSK. Both MSK and N-KVC have financial interest in YmAbs, Abpro-Labs and Eureka Therapeutics. N-KVC reports receiving commercial research grants from Y-mabs Therapeutics and Abpro-Labs Inc. N-KVC was named as inventor on multiple patents filed by MSK, including those licensed to Ymabs Therapeutics, Biotec Pharmacon, and Abpro-labs. N-KVC is an SAB member for Eureka Therapeutics.

Patient consent for publication Not applicable.

Ethics approval All animal experiments were approved by the Memorial Sloan Kettering's Institutional Animal Care and Use Committee (IACUC) and were executed according to the ACUC guidelines. Patient-derived tumor xenografts were established with MSKCC IRB approval.

Provenance and peer review Not commissioned; externally peer reviewed.

Data availability statement Data are available in a public, open access repository.

Supplemental material This content has been supplied by the author(s). It has not been vetted by BMJ Publishing Group Limited (BMJ) and may not have been peer-reviewed. Any opinions or recommendations discussed are solely those of the author(s) and are not endorsed by BMJ. BMJ disclaims all liability and responsibility arising from any reliance placed on the content. Where the content includes any translated material, BMJ does not warrant the accuracy and reliability of the translations (including but not limited to local regulations, clinical guidelines, terminology, drug names and drug dosages), and is not responsible for any error and/or omissions arising from translation and adaptation or otherwise.

Open access This is an open access article distributed in accordance with the Creative Commons Attribution Non Commercial (CC BY-NC 4.0) license, which permits others to distribute, remix, adapt, build upon this work non-commercially, and license their derivative works on different terms, provided the original work is properly cited, appropriate credit is given, any changes made indicated, and the use is non-commercial. See http://creativecommons.org/licenses/by-nc/4.0/.

\section{ORCID iDs}

Jeong A Park http://orcid.org/0000-0003-3690-6747

Nai-Kong V Cheung http://orcid.org/0000-0001-6323-5171

\section{REFERENCES}

1 Ellerman D. Bispecific T-cell engagers: towards understanding variables influencing the in vitro potency and tumor selectivity and their modulation to enhance their efficacy and safety. Methods 2019;154:102-17.

2 Wagner J, Wickman E, DeRenzo C, et al. Car T cell therapy for solid tumors: bright future or dark reality? Molecular Therapy 2020;28:2320-39. 
3 Shah NN, Fry TJ. Mechanisms of resistance to CAR T cell therapy. Nat Rev Clin Oncol 2019;16:372-85.

4 Martinez M, Moon EK. Car T cells for solid tumors: new strategies for finding, infiltrating, and surviving in the tumor microenvironment. Front Immunol 2019;10:128

5 Shah NN, Maatman T, Hari P, et al. Multi targeted CAR-T cell therapies for B-cell malignancies. Front Oncol 2019;9:146.

6 Kantarjian H, Stein A, Gökbuget N, et al. Blinatumomab versus chemotherapy for advanced acute lymphoblastic leukemia. $N$ Engl $J$ Med 2017;376:836-47.

7 Offner S, Hofmeister R, Romaniuk A, et al. Induction of regular cytolytic $\mathrm{T}$ cell synapses by bispecific single-chain antibody constructs on $\mathrm{MHC}$ class I-negative tumor cells. Mol Immunol 2006;43:763-71.

8 Kebenko M, Goebeler M-E, Wolf M. A multicenter phase 1 study of solitomab (MT110, AMG 110), a bispecific EpCAM/CD3 T-cell engager $(B \mathrm{BTE} \otimes)$ antibody construct, in patients with refractory solid tumors. Oncoimmunology 2018;7:e1450710.

9 Lum LG, Thakur A, Al-Kadhimi Z, et al. Targeted T-cell therapy in stage IV breast cancer: a phase I clinical trial. Clin Cancer Res 2015;21:2305-14.

10 Vaishampayan U, Thakur A, Rathore R, et al. Phase I study of antiCD3 $\mathrm{X}$ anti-HER2 bispecific antibody in metastatic castrate resistant prostate cancer patients. Prostate Cancer 2015;2015:285193

11 Reusch U, Sundaram M, Davol PA, et al. Anti-Cd3 X anti-epidermal growth factor receptor (EGFR) bispecific antibody redirects T-cell cytolytic activity to EGFR-positive cancers in vitro and in an animal model. Clin Cancer Res 2006;12:183-90.

12 Lum LG, Thakur A, Pray C, et al. Multiple infusions of CD20-targeted T cells and low-dose IL-2 after SCT for high-risk non-Hodgkin's lymphoma: A pilot study. Bone Marrow Transplant 2014;49:73-9.

13 Park JA, Santich $\mathrm{BH}, \mathrm{Xu} \mathrm{H}$, et al. Potent ex vivo armed T cells using recombinant bispecific antibodies for adoptive immunotherapy with reduced cytokine release. J Immunother Cancer 2021;9:e002222.

14 Park JA, Cheung N-KV. Gd2 or HER2 targeting T cell engaging bispecific antibodies to treat osteosarcoma. $J$ Hematol Oncol 2020;13:172.

15 Santich BH, Park JA, Tran H, et al. Interdomain spacing and spatial configuration drive the potency of IgG-[L]-scFv T cell bispecific antibodies. Sci Transl Med 2020;12.

16 Lin T-Y, Park JA, Long A, et al. Novel potent anti-STEAP1 bispecific antibody to redirect T cells for cancer immunotherapy. J Immunother Cancer 2021;9:e003114

17 Hoseini SS, Guo H, Wu Z, et al. A potent tetravalent T-cell-engaging bispecific antibody against CD33 in acute myeloid leukemia. Blood Adv 2018;2:1250-8.

$18 \mathrm{Wu}$ Z, Guo H-F, Xu H, et al. Development of a tetravalent AntiGPA33/Anti-CD3 bispecific antibody for colorectal cancers. Mol Cancer Ther 2018;17:2164-75.

$19 \mathrm{Xu} \mathrm{H}$, Cheng M, Guo H, et al. Retargeting T cells to GD2 pentasaccharide on human tumors using bispecific humanized antibody. Cancer Immunol Res 2015;3:266-77.

20 Lopez-Albaitero A, Xu H, Guo H, et al. Overcoming resistance to HER2-targeted therapy with a novel HER2/CD3 bispecific antibody. Oncoimmunology 2017;6:e1267891.

21 Orcutt KD, Ackerman ME, Cieslewicz M, et al. A modular IgG-scFv bispecific antibody topology. Protein Eng Des Sel 2010;23:221-8.

22 Reikofski J, Tao BY. Polymerase chain reaction (PCR) techniques for site-directed mutagenesis. Biotechnol Adv 1992;10:535-47.

23 Andrade D, Redecha PB, Vukelic M, et al. Engraftment of peripheral blood mononuclear cells from systemic lupus erythematosus and antiphospholipid syndrome patient donors into BALB-RAG-2-/IL-2R $\gamma-/-$ mice: a promising model for studying human disease. Arthritis \& Rheumatism 2011;63:2764-73.
24 Stone JD, Artyomov MN, Chervin AS. Interaction of StreptavidinBased Peptide-MHC oligomers (tetramers) with cell-surface TCRs 2011;187:6281-90.

25 Sterner RC, Sterner RM. Car-T cell therapy: current limitations and potential strategies. Blood Cancer J 2021;11:69.

26 Han X, Wang Y, Wei J, et al. Multi-antigen-targeted chimeric antigen receptor T cells for cancer therapy. J Hematol Oncol 2019;12:128.

27 Hegde M, Corder A, Chow KKH, et al. Combinational targeting offsets antigen escape and enhances effector functions of adoptively transferred T cells in glioblastoma. Mol Ther 2013;21:2087-101.

28 Hegde M, Mukherjee M, Grada Z, et al. Tandem CAR T cells targeting HER2 and IL13R 2 mitigate tumor antigen escape. J Clin Invest 2016;126:3036-52.

29 Tong C, Zhang Y, Liu Y, et al. Optimized tandem CD19/CD20 CARengineered T cells in refractory/relapsed B-cell lymphoma. Blood 2020;136:1632-44.

30 Schneider D, Xiong Y, Wu D, et al. Trispecific CD19-CD20-CD22targeting duoCAR-T cells eliminate antigen-heterogeneous $B$ cell tumors in preclinical models. Sci Trans/ Med 2021;13 doi:10.1126/ scitranslmed.abc6401

31 Hwang MS, Mog BJ, Douglass J, et al. Targeting loss of heterozygosity for cancer-specific immunotherapy. Proc Natl Acad Sci U S A 2021;118:e2022410118.

32 Maude SL, Frey N, Shaw PA, et al. Chimeric antigen receptor T cells for sustained remissions in leukemia. N Engl $\mathrm{J} \mathrm{Med}$ 2014;371:1507-17.

33 Fry TJ, Shah NN, Orentas RJ, et al. CD22-targeted CAR T cells induce remission in B-ALL that is naive or resistant to CD19-targeted CAR immunotherapy. Nat Med 2018;24:20-8.

34 Watanabe K, Terakura S, Martens AC. Target antigen density governs the efficacy of anti-CD20-CD28-CD3 $\zeta$ chimeric antigen receptormodified effector CD8 ${ }^{+}$T cells 2015;194:911-20.

35 Caruso HG, Hurton LV, Najiar A, et al. Tuning sensitivity of car to EGFR density limits recognition of normal tissue while maintaining potent antitumor activity. Cancer Res 2015;75:3505-18.

36 Zhao J, Song Y, Liu D. Clinical trials of dual-target CAR T cells, donor-derived CAR T cells, and universal CAR T cells for acute lymphoid leukemia. J Hematol Oncol 2019;12:17.

37 Chmielewski M, Hombach A, Heuser C, et al. T cell activation by antibody-like immunoreceptors: increase in affinity of the singlechain fragment domain above threshold does not increase T cell activation against antigen-positive target cells but decreases selectivity. J Immunol 2004;173:7647-53.

38 Park S, Shevlin E, Vedvyas $\mathrm{Y}$, et al. Micromolar affinity CAR T cells to ICAM-1 achieves rapid tumor elimination while avoiding systemic toxicity. Sci Rep 2017;7:14366.

39 Watanabe K, Kuramitsu S, Posey AD, et al. Expanding the therapeutic window for CAR T cell therapy in solid tumors: the knowns and unknowns of CAR T cell biology. Front Immunol 2018;9:2486.

40 Csizmar CM, Petersburg JR, Perry TJ, et al. Multivalent ligand binding to cell membrane antigens: defining the interplay of affinity, valency, and expression density. J Am Chem Soc 2019;141:251-61.

41 Park JA, Wang L, Cheung N-KV. Modulating tumor infiltrating myeloid cells to enhance bispecific antibody-driven T cell infiltration and antitumor response. J Hematol Oncol 2021;14:142.

42 Walrath JC, Hawes JJ, Van Dyke T, et al. Genetically engineered mouse models in cancer research. Adv Cancer Res 2010;106:113-64.

43 Zilionis R, Engblom C, Pfirschke C, et al. Single-Cell transcriptomics of human and mouse lung cancers reveals conserved myeloid populations across individuals and species. Immunity2019;50:1317-34. 